Document downloaded from:

http://hdl.handle.net/10251/64595

This paper must be cited as:

Bravo, R.; Pérez Aparicio, JL.; Gómez Hernández, JJ. (2015). Numerical sedimentation particle-size analysis using the Discrete Element Method. Advances in Water Resources. 86:58-72. doi:10.1016/j.advwatres.2015.09.024.

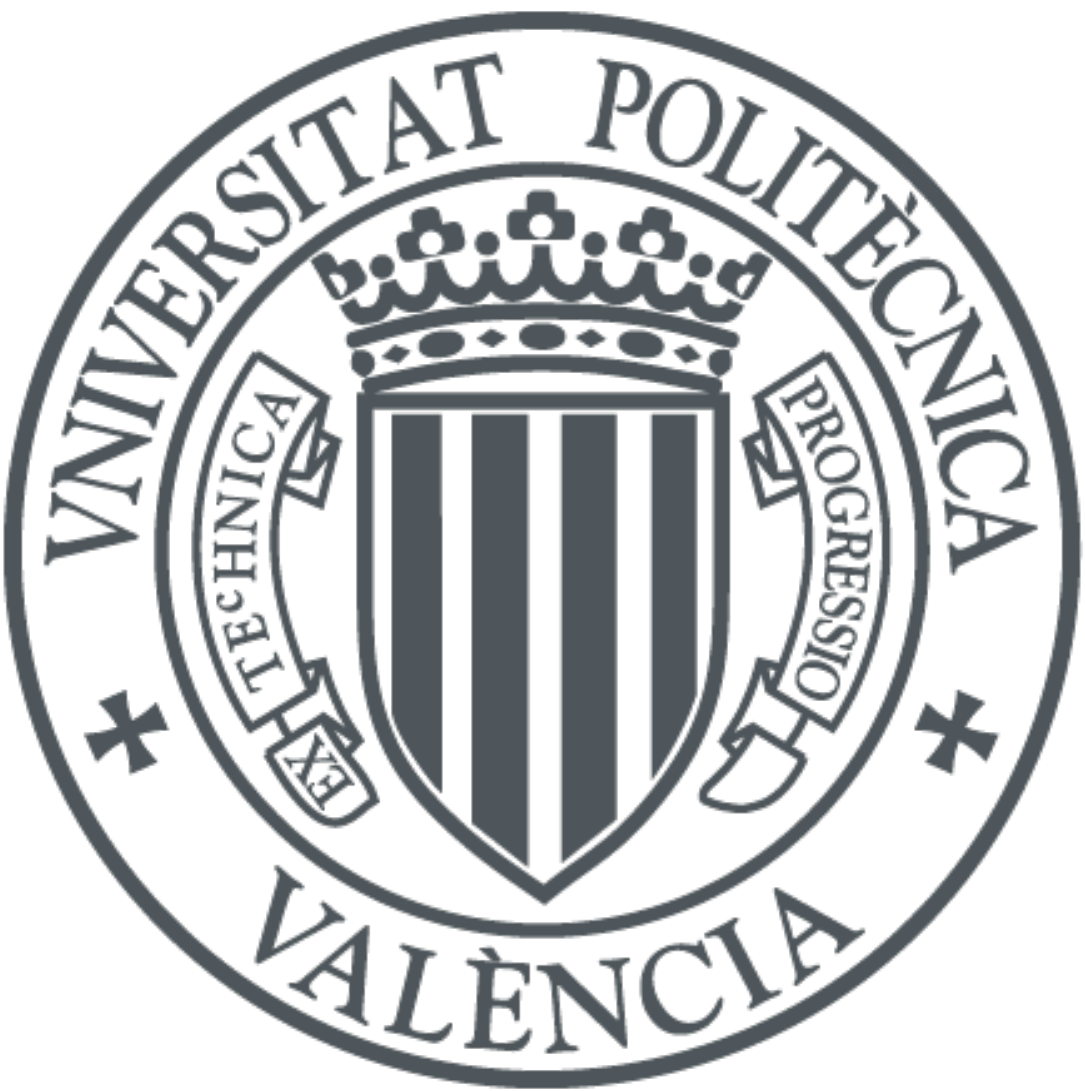

The final publication is available at

http://dx.doi.org/10.1016/j.advwatres.2015.09.024

Copyright Elsevier

Additional Information 


\title{
Numerical sedimentation particle-size analysis using the Discrete Element Method
}

\author{
R. Bravo ${ }^{\mathrm{a}, *}$, J.L. Pérez-Aparicio ${ }^{\mathrm{b}}$, J.J. Gómez-Hernández ${ }^{\mathrm{c}}$ \\ ${ }^{a}$ Department of Structural Mechanics and Hydraulic Engineering, University of Granada 18071 Granada, Spain \\ ${ }^{b}$ Department of Continuum Mechanics and Theory of Structures, Universitat Politècnica de València 46022 Valencia, Spain \\ ${ }^{c}$ Research Institute of Water and Environmental Engineering, Universitat Politècnica de València 46022 Valencia, Spain
}

\begin{abstract}
Sedimentation processes are generally modelled using an equivalent continuum approach based on the coupling of the Navier-Stokes and the advection-dispersion equations; the sediment concentration within an elementary fluid volume is the variable of interest. Continuous advances in computational capabilities have brought up a new type of modelling that simulates the individual motion and contact interactions of grains: the Discrete Element Method (DEM). In this work, DEM interacts with the simulation of flow using the well known one-way-coupling method, a computationally affordable approach for the time-consuming numerical simulation of the ASTM-D422, buoyancy and pipette sedimentation tests. These tests are used in the laboratory to determine the particle-size distribution of fine-grained aggregates.

Five samples with different particle-size distributions are modelled by about six million rigid spheres projected on two-dimensions, with diameters ranging from $2.5 \times 10^{-6} \mathrm{~m}$ to $70 \times 10^{-6} \mathrm{~m}$, forming a water suspension in a sedimentation cylinder. DEM simulates the particle's movement considering laminar flow interactions of hydrostatic thrust, drag and lubrication forces. The simulation provides the temporal/spatial distributions of densities and concentrations of the suspension.

The numerical simulation cannot replace the laboratory tests since it needs the final granulometry as initial data; but, as the results show, these simulations can identify the strong and weak points of each method and eventually recommend useful variations and draw conclusions on their validity, aspects very difficult to achieve in the laboratory.
\end{abstract}

Keywords: ASTM-D-422, buoyancy pipette sedimentation tests, particle size distribution, discrete element method, drag-lubrication forces

\section{Nomenclature}

List of symbols with sub- and supra-indices by order of appearance in the article. The units of these symbols are in the base-unit SI system.

\section{Introduction}

Numerical continuum approaches have traditionally been employed to simulate the suspension sedimentation of a large number (millions) of particles in a flow. The concentration evolution is often analyzed using advectiondispersion equations (see $[1,2,3,4]$ ). The traditional continuum approach (see Fig. 1, right top) is a simplification of reality, that nonetheless, has provided good results in many applications $[5,6,7,8]$; however, the real physics of the problem is better represented by individual particles transported by a fluid, with flow interacting with the particles that, in turn, interact among themselves.

\footnotetext{
${ }^{*}$ Corresponding author. Tel.: +34 958249960

Email addresses: rbravo@ugr.es (R. Bravo), jopeap@upvnet.upv.es (J.L. Pérez-Aparicio), jaime@dihma.upv.es (J.J. Gómez-Hernández)
}

In this work, the granular media is analyzed as a discontinuous structure with rigid particles and voids between them, see Fig. 1, right bottom. The formulation of the Discrete Element Method (DEM) [9] probably is the most suitable to simulate the behavior of granular materials in these situations, since it is based on the study of the individual particles and their contact interactions. Additionally, DEM can capture other information such as arrangements of particles or global constitutive laws (contact algorithms in this context, see [10]), aspects very difficult to account for with numerical methods based on continuum media.

Nowadays, and thanks to the increased computational resources, it is possible to conceive the modelling of the sedimentation of a large number of particles by addressing the physics of the problem at -or approximately- the grain scale. Next, we revise some of the methods that have been developed to analyze the particle-fluid interaction and motion of particles inside a fluid.

Lagrangian computational fluid dynamics (CFD) methods are based on multiphase continuum techniques, see [11], and are applied to analyze the behaviour of sprays, small bubbles and dust particles; processes in which the contact interactions are negligible. The Immersed Bound- 


\begin{tabular}{|c|c|}
\hline Symbl & Description \\
\hline$g$ & Gap \\
\hline$N, N$ & Normal vector or direction \\
\hline$X, x, X$ & Horizontal position/coordinate \\
\hline $\boldsymbol{Y}, y, Y$ & Vertical position/coordinate \\
\hline$T, T$ & Tangential vector/direction \\
\hline$n$ & Number, index \\
\hline $\boldsymbol{U}, \boldsymbol{u}, U$ & Displacement \\
\hline$t$ & Time \\
\hline$f$ & Force \\
\hline$K$ & Penalty stiffness \\
\hline$m$ & Mass \\
\hline$w$ & Weight \\
\hline$I$ & Inertia \\
\hline$M$ & Moment \\
\hline$w$ & $\%$ accumulated weight \\
\hline $\operatorname{Re}$ & Reynolds number \\
\hline$C$ & Coefficient \\
\hline$A$ & Area \\
\hline$c$ & Concentration \\
\hline$e$ & Empirical exponent \\
\hline$r$ & Aspect ratio \\
\hline$g$ & Gravity acceleration \\
\hline$v$ & Volume \\
\hline$b, B$ & Width \\
\hline$h, H$ & Height \\
\hline$p$ & Pressure \\
\hline$j$ & Unit vertical vector \\
\hline$\Delta$ & Increment \\
\hline$\Phi$ & Frictional Coulomb's law \\
\hline$\mu$ & Friction coefficient \\
\hline$\varphi$ & Friction angle \\
\hline$\theta$ & Angular rotation \\
\hline$\phi$ & Diameter \\
\hline$\rho$ & Density \\
\hline$v$ & Dynamic viscosity \\
\hline
\end{tabular}

ary Method, see [12], simulates the fluid-particle interactions: fluid and structure are represented by Eulerian and Lagrangian coordinates, respectively. In turn, the NavierStokes equations are solved in a structured grid so that the effort needed to generate a body-fitted grid is avoided, and, at the same time, body resistant forces are obtained. The method requires an accurate discretization of particles and fluid, therefore, its application is limited to problems with a small number of particles. Stokesian Dynamics, see $[13,14]$, simulates the flow and particles subjected to stochastic Brownian forces, but again requires a high computational cost for a relatively small number of particles, see $[15]$.

The computational cost for a large number of particles was recently reduced by the combination of DEM and CFD, see [16]. In the CFD-DEM models of fluid-particles systems, the solid motion is obtained by DEM but the flow motion is described by locally averaged Navier-Stokes equations solved using a CFD approach. The coupling be-

\begin{tabular}{|c|c|}
\hline Symbol & Sub- or supra-index \\
\hline$\overline{(0)^{i, k}}$ & Particle number indices \\
\hline$(0)_{t t}$ & Total \\
\hline$(0)_{c}$ & Contact \\
\hline (ö) & Second-time derivative \\
\hline (०) $c g$ & Center of Gravity \\
\hline (०) $)_{d}^{g}$ & Drag \\
\hline (०) $)_{l}$ & Lubrication \\
\hline (०) $)_{h}$ & Hydrostatic \\
\hline (ं) & First-time derivative \\
\hline$(0)_{o}$ & Ordered \\
\hline (०) $h d$ & Hydrometer \\
\hline$(0)_{b t}$ & Buoyancy test \\
\hline (o) $p t$ & Pipette test \\
\hline (०) $f$ & Fluid \\
\hline (o) $t r$ & Terminal \\
\hline$(0)_{S t}$ & Stokes \\
\hline$(0)_{p}$ & Particle \\
\hline$(\mathrm{o})^{l f}$ & Left \\
\hline$(0)^{r t}$ & Right \\
\hline (o) $)_{c y}$ & Cylinder \\
\hline (०) $l y$ & Layer of cylinder \\
\hline (०) $)_{j h}$ & Layer in contact $\mathrm{w} / \mathrm{bulb}$ \\
\hline (०) $c o$ & Column \\
\hline$(0)_{s}$ & Suspension \\
\hline$(0)_{z}$ & Out-of-plane dimension \\
\hline$(\circ)_{0}$ & Initial \\
\hline$(0)_{w}$ & Pure water \\
\hline (o) $s p$ & Buoyancy test sphere \\
\hline$(0)_{a}$ & Pure air \\
\hline (०) $)_{d e m}$ & Discrete element method \\
\hline$(\mathrm{o})_{t s t}$ & Experimental test \\
\hline
\end{tabular}

tween fluid and solid phases is modelled by Newton's third law.

Finally, the coupling of the Lattice-Boltzmann method with DEM, see [17], is also suitable to simulate motion of particles in a flow but requires an accurate and refined discretization, not computationally suitable for the problems studied here as explained in [18].

In this work, flow and particles are coupled using the one-way-coupling method, see [19]. This technique substantially reduces computation, considering that flow does modify particle movement but particles do not substantially modify flow: see [20] for the application of this technique to the analysis of inclined sediment beds. The assumption is acceptable to simulate sedimentation of finegrained materials due to the small size and low deposition velocities of the particles. In addition, the action of the flow is analytically represented by the flow-particle interaction modelled by the drag force taking into account concentration of the neighboring particles, see [21], and by the squeezing of the intermediate flow when two particles are close, see [22, 23]. These two models permit the calculation of forces other than the contact forces that are included into DEM, allowing a good representation 


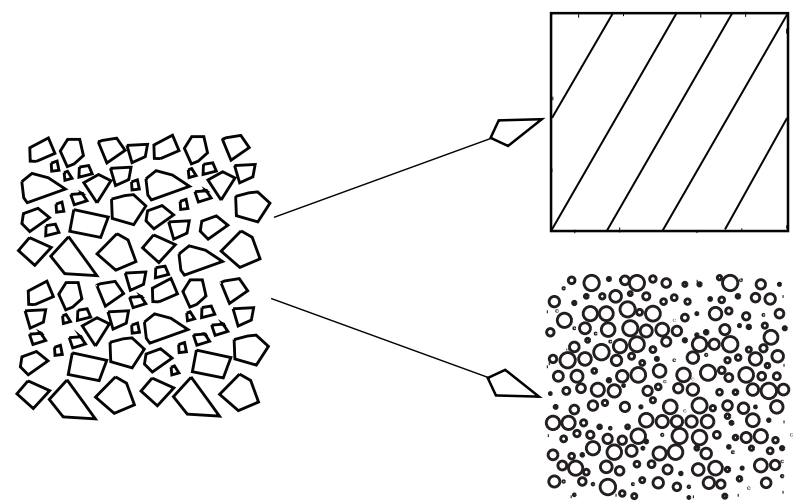

Figure 1: Granular medium (left). Equivalent continuum medium model (top right) and discontinuous medium modelled with $2 \mathrm{D}$ projected spheres (bottom right).

of the long time deposition process at an affordable CPU cost. Due to the dissipative character of the drag forces, the equations are solved using the time integration scheme from [24], which offers a stable and physically consistent framework to model dissipation phenomena. Finally, the contact interactions between particles are simulated using the penalization technique from [25].

This paper is centered on the numerical simulation of the ASTM D-422 [26] sedimentation test; in addition and for comparison purposes the less used buoyancy, [27, 28], and pipette [29] tests are also studied. The three tests are used in the laboratory to determine the particle size distribution of granular samples with diameters ranging from $2.5 \times 10^{-6} \mathrm{~m}$ to $70 \times 10^{-6} \mathrm{~m}$; distributions that cannot be easily measured by standard sieving methods as explained in [30]. DEM incorporates the particle kinematics and therefore the temporal and spatial distributions of densities and concentrations of the suspension. These data along with the laws of hydrostatics are used to numerically reproduce the measurements of a hydrometer 152-H, a buoyancy sphere and a pipette.

The target of the work is to numerically replicate the experiments, providing a better understanding of the sedimentation and of the data collected from the three tests. A comparison of the results derived by the numerical simulation of the laboratory experiments to the five original a-priori known distributions is performed with good agreement, validating the simulations and identifying several issues regarding the interpretation of the laboratory observations.

Although tests based in sedimentation are widely used due to their simplicity and relatively low cost, it is important to remark that other expensive and sophisticated techniques have been developed for the particle size analysis using x-rays, see [31] or a combination of x-rays and centrifugation, see [32].

The remaining of the paper has the following structure: Sect. 3 introduces the DEM method, its formulation and the terms necessary for the one-way coupling of the particle-flow interaction. Section 4 analyzes the sed- imentation test, and describes its numerical implementation, including the forces acting on the particles and how to project the real axisymmetric geometry into a twodimensional (2D) one. Finally, Sect. 5 presents and analyzes the results of the numerical simulations, with discussions on the accuracy of the laboratory experiments and on the quality of the measurement techniques.

\section{Discrete element method}

DEM is a numerical tool that naturally simulates the behaviour of particle materials. It is able to model the geometry of each particle and to capture the mechanical response without the necessity of complex constitutive laws; the global response of a set of particles is a consequence of multiple contact/friction interactions among a large number of particles (micro-mechanics). Multiple simultaneous and long-lasting contacts might be produced generating particle clusters; an appropriate technique to simulate the contact interaction among particles is penalization, see [25]. This technique does not increase the number of unknowns and produces only small particle overlaps, see Fig. 2 left. In addition, these unphysical overlaps are minimized by a proper tuning of the numerical parameters used in the penalization approach.

The DEM detects contacts between particles through a gap function $g_{N}^{i k}$ that measures the minimum distance or maximum overlap between two particles numbered $i$ and $k$, as

$$
g_{N}^{i k}=(\boldsymbol{X}-\boldsymbol{Y}) \boldsymbol{N}^{i k}
$$

where $\boldsymbol{X}, \boldsymbol{Y}$ are the coordinates of the two points, one in each particle, that give the minimum distance (if particles are not in contact) or the maximum penetration (if they overlap). The penetration, when happens, is always minimal, although it has been exaggerated in Fig. 2 for clarity. When this penetration occurs, or if the gap function is zero, we will say that the particles are in contact. $\boldsymbol{N}^{i k}=-\boldsymbol{N}^{k i}$ is the normal unit vector at the contact point, see Fig. 2.

In case of contact, the relative motion between the contact points in the tangential component is measured by the tangential gap $g_{T}^{i k}$ as

$$
g_{T}^{i k}=\left[\boldsymbol{X}+\boldsymbol{U}^{i}(\boldsymbol{X})-\boldsymbol{Y}-\boldsymbol{U}^{k}(\boldsymbol{Y})\right] \boldsymbol{T}^{i k},
$$

where $\boldsymbol{U}^{i}(\boldsymbol{X})$ and $\boldsymbol{U}^{k}(\boldsymbol{Y})$ are the displacements of the contact points of $i$ and $k$ corresponding to a time increment $\Delta t$, while $\boldsymbol{T}^{i k}$ is the tangential unit vector at the contact point as in Fig. 2.

The contact particle interactions are mechanically modelled by defining the contact force as

$$
\boldsymbol{f}_{c}^{i k}=f_{c N}^{i k} \boldsymbol{N}^{i k}+f_{c T}^{i k} \boldsymbol{T}^{i k},
$$

where $f_{c N}^{i k}$ and $f_{c T}^{i k}$ are the components of the contact force that act against the interpenetration in the normal direction and control the motion in the tangential direction, 


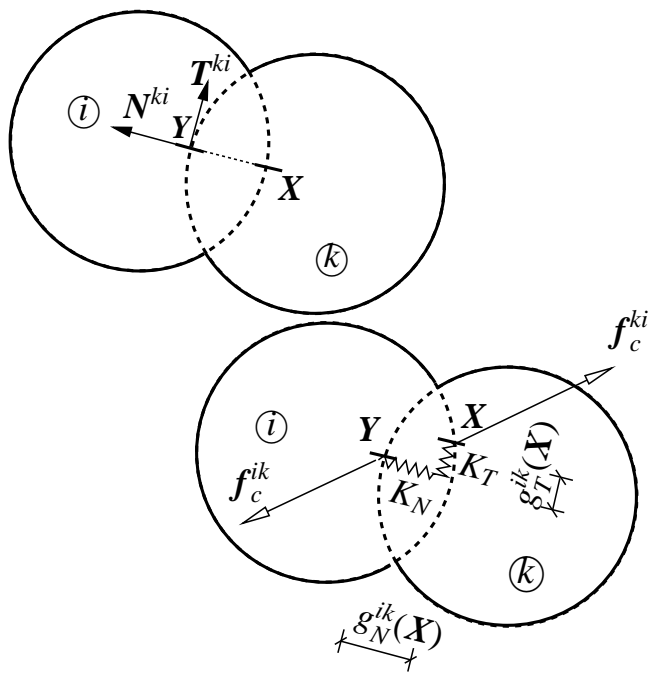

Figure 2: Top: Penetrating contact between two particles. Bottom: $g_{N}^{i k}$ defines maximum penetration, $g_{T}^{i k}$ tangential displacement. Overlapping magnified for better representation.

respectively. For spherical and rigid particles the contact can be represented by a point force applied at a single point. Defining

$$
\Phi^{i k}=f_{c T}^{i k}-\mu\left|f_{c N}^{i k}\right|
$$

where, according to Coulomb's law $\mu\left|f_{c N}^{i k}\right|$ is the maximum friction force, with $\mu=\tan \varphi$ being the friction coefficient, and $\varphi$, the friction angle; we can distinguish between motion by rolling if $\Phi^{i k}<0$ (lack of relative motion at the contact point) or by sliding if $\Phi^{i k} \geq 0$.

The penalization technique used to determine the contact forces between the two particles consists on introducing two virtual high stiffness elastic springs at the contact points $\boldsymbol{X}, \boldsymbol{Y}$ along the normal and tangential directions (see Fig. 2 right) resulting in: $f_{c N}^{i k}=K_{N} g_{N}^{i k}$ and $f_{c T}^{i k}=K_{T} g_{T}^{i k}$ for rolling, or $f_{c N}^{i k}=K_{N} g_{N}^{i k}$ and $f_{c T}^{i k}=\mu f_{c N}^{i k}$ for sliding. The $K_{N}$, $K_{T}$ and $\mu$ coefficients are the constitutive parameters of the model, the first two being artificial numerical parameters representing the stiffnesses of the elastic springs. These stiffnesses must be chosen according to the prescriptions given in [33].

The governing equations of DEM for a system of $n_{t t}$ particles are formulated by the translational and rotational dynamic equilibrium (see Fig. 3) of each particle with mass $m^{i}$ and inertia $I^{i}$

$$
m^{i} \ddot{\boldsymbol{u}}^{i}=\boldsymbol{f}_{c}^{i}+\boldsymbol{f}_{d}^{i}+\boldsymbol{f}_{l}^{i}+\boldsymbol{f}_{h}^{i}+\boldsymbol{w}^{i} ; \quad I^{i} \ddot{\theta}^{i}=\boldsymbol{M}^{i},
$$

where $\boldsymbol{u}^{i}=\left\{u_{x}^{i}, u_{y}^{i}\right\}$ and $\theta^{i}$ are the displacement of and rotation around the center of gravity $(c g)$ of each particle; the supraindex ( " ) denotes second time derivative. The force $\boldsymbol{f}_{c}^{i}=\sum_{k=1}^{n_{t t}} \boldsymbol{f}_{c}^{i k}$ is the vectorial resultant of the contact forces of all other particles acting on particle $i$; the rest of forces $\boldsymbol{f}_{d}^{i}, \boldsymbol{f}_{l}^{i}, \boldsymbol{f}_{h}^{i}, \boldsymbol{w}^{i}$ are the drag, lubrication, hydrostatic and weight, all of them applied at the $c g$ of $i$ and defined in the next section. In the second equation $\boldsymbol{M}^{i}$ is the only non-zero moment generated by the friction contact forces: the other forces do not create moments for spherical particles.

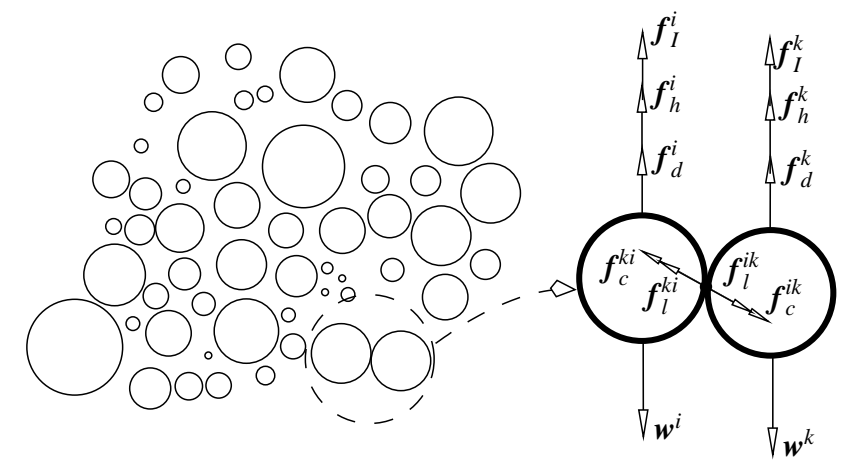

Figure 3: Forces on spheres sedimenting inside a viscous fluid.

In 2D, Eqs. (5) form a set of $3 n_{t t}$ non-linear equations with a right-hand side dependent on the displacement $\boldsymbol{u}^{i}$ through force $\boldsymbol{f}_{c}^{i}$ and on velocity $\dot{\boldsymbol{u}}^{i}$ through $\boldsymbol{f}_{d}^{i}$. The left hand sides inertial terms depend on the two unknowns, translational and rotational accelerations $\ddot{\boldsymbol{u}}^{i}$ and $\ddot{\theta}^{i}$.

The displacements $U^{i}$ of any point $\left(x^{i}, y^{i}\right)$ within $i$ are obtained by first order interpolation using

$$
\boldsymbol{U}^{i}=\left\{\begin{array}{c}
U_{x}^{i} \\
U_{y}^{i}
\end{array}\right\}=\left[\begin{array}{rrr}
1 & 0 & -\left(y^{i}-y_{c g}^{i}\right) \\
0 & 1 & \left(x^{i}-x_{c g}^{i}\right)
\end{array}\right]\left\{\begin{array}{c}
u_{x}^{i} \\
u_{y}^{i} \\
\theta^{i}
\end{array}\right\},
$$

where $x_{c g}^{i}, y_{c g}^{i}$ are the coordinates of the $c g$ of $i$. Finally, the time integration of Eqs. (5) is carried out by an implicit single-step algorithm proposed in $[34,24]$. The choice is based on the algorithm capability to simulate the dissipation produced by friction and drag forces in a physically consistent manner.

\section{The sedimentation tests}

\subsection{Description}

This experiment is traditionally performed to obtain the grading size distribution of samples with particles of diameters of less than $70 \times 10^{-6} \mathrm{~m}$. The standard ASTM-D422 describes the procedure to obtain the grading size distribution using the sedimentation test: a sample of total solid (particles) mass $m_{t t}$ of $60 \times 10^{-3} \mathrm{Kg}$ is poured into a cylinder full of water. The cylinder is shaken during $60 \mathrm{~s}$, so that the particles are dispersed forming a suspension with homogeneous concentration. Then, the cylinder rests in vertical position and the particles start sinking with velocities dependent to their sizes. The concentration of the suspension progressively gradates in the vertical direction as a function of the particles' sizes. After several hours, most of the large and intermediate particles settle at the cylinder bottom, while the finer particles are still at the top and middle. 
ASTM-D422 provides the grading size distribution at different instants through the measurements of a hydrometer, composed of bulb and stem. The hydrometer immersed in a suspension measures the concentration around the bulb, which sinks a certain depth. As particles concentrate towards the bottom, the hydrometer gradually sinks deeper since the concentration around the bulb reduces. The depth of the hydrometer measured from the bulb center to the water surface taken at different instants provides and indirect measurement of the time variation of fluid density (and consequently of particle concentrations) at the bulb level; depth is related to fluid density using the analytical laws of flotation from [35].

The buoyancy and pipette tests provide the grading size distribution differently, measuring the concentration of the suspension at different instants but at a fixed depth.

The buoyancy test measures the suspension concentration at a specific and constant depth $y_{b t}$ as described in [27]. A Teflon sphere of diameter $2.5 \times 10^{-2} \mathrm{~m}$ and weight $20 \times 10^{-3} \mathrm{Kg}$ attached to a nylon line connected to a sensitive scale is submerged into the liquid. The scale measures the apparent mass of the sphere.

The pipette test is conceptually the most simple and accurate test, see [29], although requires the additional use of a drier and a precision scale. At prescribed times, particle concentrations are measured by weighing the dry residual resulting from $35 \mathrm{ml}$ samples taken at a specified and constant depth $y_{p t}$ using a standard pipette.

In the three tests, limiting diameters and accumulated weights are determined (as it will be explained later) up to ten times, and with them an approximation of the particle size distribution is obtained. The limiting diameter $\phi_{o}$ is the maximum diameter of the particles above a certain depth. The accumulated weight $w_{o}$ is the weight of all particles with a diameter smaller than $\phi_{o}$. The pairs $\phi_{o}, w_{o}$ observed at different times provide the particle size distribution, commonly called granulometry of the sample.

The target of the numerical simulation described in this section (and to the best of our knowledge not published by other authors) is to numerically replicate the physics of the three sedimentation tests: hydrometer ASTM D422, buoyancy and pipette. The numerical simulations start with a given grading size distribution and simulates the sedimentation providing the position, velocities and accelerations of all particles at all instants. From these results, it is relatively simple to reproduce the measurements as they would have been observed during each of the three tests. The grain distributions resulting from the numericallyreproduced test measurements differ only slightly from the starting one, indicating that the experiments may not yield an exact granulometry of the sample.

The remaining of this section describes the physics and the numerical implementation of the experiments.

\subsection{Fluid-particle interaction}

The experiments simulated in this work include particles with small Reynolds numbers, more precisely, the
Reynolds numbers for the particles in all samples analyzed range from $1.2 \times 10^{-5}$ to 0.28 as determined from the observed minimum and maximum terminal velocities. Therefore, the flow is laminar, a consequence of the small size of the particles and their small depositional velocity. We can assume that particles do not perturb the fluid, although the fluid does affect the particles as they move. This "one-way coupling" is a computationally affordable assumption that allows the simulation of the interactions between fluid and particles. The action of the fluid on the particles is represented by the Stokes' drag force, $\boldsymbol{f}_{d}^{i}$, and by the squeezing force $\boldsymbol{f}_{l}^{i}$, which appear in the right hand side of Eqs. (5). Their expressions are derived next.

\subsubsection{Stokes' drag forces}

Forces created by fluid-particle interaction have traditionally been modelled using Stokes' law, see [36], a simplified model that does not take into account the interactions of other particles in the surrounding. This law is not completely valid to study sedimentation, but provides the basis for the analytical formulation of the ASTM D-422, buoyancy and pipette tests. For the numerical modeling, the more sophisticated formulation from [21] solves the previous deficiency, resulting in the $\operatorname{drag}$ force $\boldsymbol{f}_{d}^{i}$ acting on $i$ as

$$
\boldsymbol{f}_{d}^{i}=\frac{1}{2} C_{d}^{i} \rho_{f} A^{i}\left|\dot{\boldsymbol{u}}_{f}^{i}-\dot{\boldsymbol{u}}^{i}\right|\left(\dot{\boldsymbol{u}}_{f}^{i}-\dot{\boldsymbol{u}}^{i}\right)\left(c^{i}\right)^{e^{i}}
$$

This expression is the one used in the numerical simulation of Eq. (5). The symbol $C_{d}^{i}$ represents the drag coefficient and depends on the particle $R e^{i}$. Both are given by

$$
C_{d}^{i}=\frac{24}{R e^{i}}\left(0.63+4.8 \sqrt{\frac{c^{i}}{R e^{i}}}\right) ; \quad R e^{i}=\frac{\rho_{f}\left|\dot{\boldsymbol{u}}^{i}-\dot{\boldsymbol{u}}_{f}^{i}\right| \phi^{i}}{v_{f}},
$$

where $\rho_{f}, v_{f}$ are the fluid density and dynamic viscosity, respectively. The remaining symbols of Eq. (7) are: $c_{i}$, particle concentration around $i, A^{i}$, area of projected particle with diameter $\phi^{i}$ into the plane perpendicular to the flow, $\dot{\boldsymbol{u}}_{f}^{i}$ velocity of the fluid around $i\left(\dot{\boldsymbol{u}}_{f}^{i} \approx 0\right.$ in this work) and $e^{i}$ is an empirically exponent given by

$$
e^{i}=-\left\{3.7-0.65 \exp \left[-\frac{1}{2}\left(1.5-\log \frac{R e^{i}}{c^{i}}\right)^{2}\right]\right\} \text {. }
$$

In the numerical simulation, the concentration $c^{i}$ of particles around $i$ is calculated by discretizing the space into a regular grid and computing the volume of particles inside each cell, see [37] and Sect. 4.4.

\subsubsection{Lubrication forces}

Each pair of approaching particles experience a repulsive lubrication force due to the squeezing of the flow between them. According to $[22,23]$, this force can be defined as

$$
\boldsymbol{f}_{l}^{i k}=10\left(r_{\phi}^{i}\right)^{3} \exp \left(\frac{-10 g_{N}^{i k}}{r_{\phi}^{i}}\right) \boldsymbol{N}^{i k}
$$


where the constant 10 is a parameter that represents the intensity and the range of the repulsive force, valid for very small spheres in water. The coefficient $r_{\phi}^{i}=2 \phi^{i} /\left(\phi^{i}+\phi^{k}\right)$ is the ratio between the particle diameter $i$ and the average diameter of the two interacting particles.

\subsection{Simple model of sedimentation}

While for the numerical modeling of sedimentation we will use the more precise expression of the drag force given by Eq. (7), the interpretation of the experiments is performed using the same simplified analytical formulation as in the sedimentation tests. We will review next this simplified model, and the procedure to infer the particle grain distribution. (Additionally, this simplified model allows us to estimate the number of time steps to be used for the numerical modeling.) The approach from [38] is based on Stokes' law for a single spherical particle, with a simplified drag force given by

$$
\left.\boldsymbol{f}_{d}^{i}\right|_{S t}=-3 \pi \phi^{i} v_{f} \dot{\boldsymbol{u}}^{i} .
$$

Now, we can establish Newton's second law for a single sphere, assuming that the only forces at which is subjected are weight, drag and buoyancy:

$$
\frac{\pi}{6}\left(\phi^{i}\right)^{3} \boldsymbol{g} \rho_{p}-\frac{\pi}{6}\left(\phi^{i}\right)^{3} \boldsymbol{g} \rho_{f}-3 \pi v_{f} \rho_{f} \phi^{i} \dot{\boldsymbol{u}}^{i}=m^{i} \ddot{\boldsymbol{u}}^{i},
$$

where $\rho_{p}$ is the particle density and $\boldsymbol{g}$ is the gravity constant. Had we included the lubrication forces or the contact forces in Eq. (12), it would have been impossible to obtain an analytical solution due to the high non-linearities introduced by these two forces. As already mention, these forces are accounted for in the numerical simulation, which should give a more accurate physical model.

Equation (13) left is the general solution of Eq. (12), for the initial condition $\dot{\boldsymbol{u}}^{i}(0)=\mathbf{0}$.

$\dot{\boldsymbol{u}}^{i}(t)=\left[1-\exp \left(-\frac{18 v_{f} t}{\rho_{p}\left(\phi^{i}\right)^{2}}\right)\right] \dot{\boldsymbol{u}}_{t r}^{i} ; \quad \dot{\boldsymbol{u}}_{t r}^{i}=\frac{\left(\rho_{p}-\rho_{f}\right) \boldsymbol{g}\left(\phi^{i}\right)^{2}}{18 v_{f}}$

The solution includes a negative exponential term that vanishes for a sufficiently long $t$, at which time the velocity reaches a constant terminal value $\dot{\boldsymbol{u}}_{t r}^{i}$. Figs. 4 (top and bottom) show sedimentation velocities computed using the previous equation for particles with density $2500 \mathrm{~kg} / \mathrm{m}^{3}$, suspended in water of density $1000 \mathrm{~kg} / \mathrm{m}^{3}$ and viscosity $10^{-3} \mathrm{~N} \cdot \mathrm{s} / \mathrm{m}^{2}$. The time to reach a $\dot{\boldsymbol{u}}^{i}=0.99 \dot{\boldsymbol{u}}_{t r}^{i}$ is found by iteratingin Eq. (13) left and it depends, quadratically, on the particle size. Terminal velocities range from $t_{t r}^{i}=3.2 \times$ $10^{-3} \mathrm{~s}$ for the largest particle to almost zero for the smallest one, with a value of $3 \times 10^{-4} \mathrm{~s}$ for an intermediate one, see also Table 1. Once the terminal velocity has been reached, the particle diameter can be estimated at a certain time directly by Eq. (13) right

$$
\phi^{i}=\sqrt{\frac{18 v_{f}}{\left(\rho_{p}-\rho_{f}\right)|\boldsymbol{g}|}} \sqrt{\left|\dot{\boldsymbol{u}}_{t r}^{i}\right|} .
$$

This expression is the one reported in the standard for the interpretation of the experiments. Notice that to derive the diameter from Eq. (14) an estimate of $\left|\dot{\boldsymbol{u}}_{t r}^{i}\right|$ is necessary; Sect. 4.5 describes how the diameters are derived.
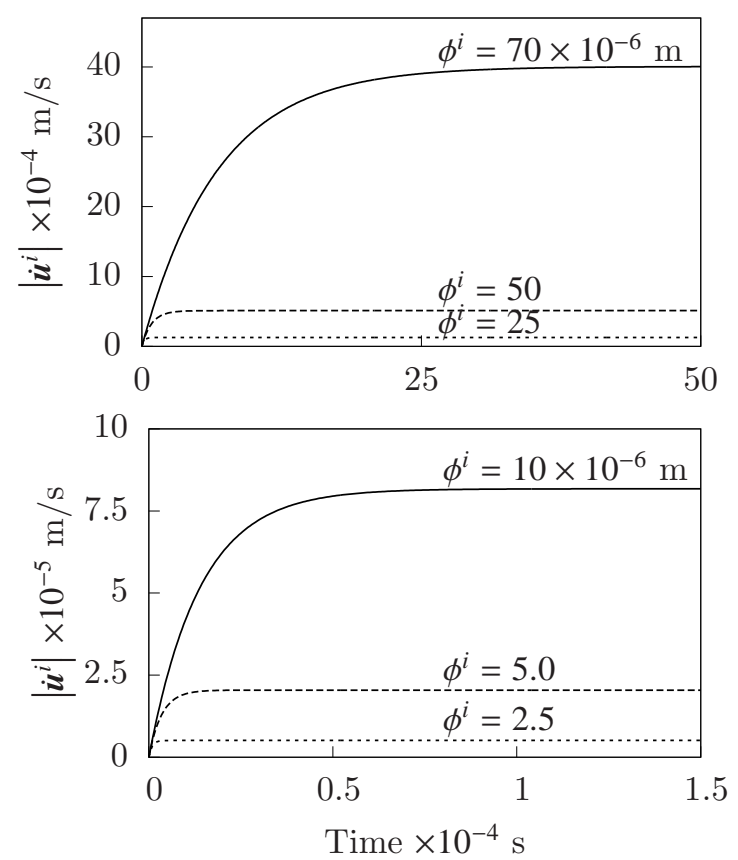

Figure 4: Particle sedimentation velocities $\left|\dot{\boldsymbol{u}}^{i}\right|$ in water as a function of time for several diameters. Notice how the terminal velocity is quickly reached.

\begin{tabular}{|c|c|c|}
\hline \hline$\phi \times 10^{-6} \mathrm{~m}$ & $t_{t r} \times 10^{-5} \mathrm{~s}$ & $\left|\dot{\boldsymbol{u}}_{t r}\right| \times 10^{-5} \mathrm{~m} / \mathrm{s}$ \\
\hline \hline 70 & 313.4 & 400.5 \\
50 & 104.0 & 204.3 \\
25 & 26.0 & 51.09 \\
10 & 4.16 & 8.17 \\
5 & 1.04 & 2.04 \\
2.5 & 0.26 & 0.51 \\
\hline \hline
\end{tabular}

Table 1: Terminal velocities $\left|\dot{\boldsymbol{u}}_{t r}\right|$ and time to reach them $t_{t r}$ derived from the approximate analytical expression for several particle diameters.

\subsection{Numerical generation of the sample}

As mentioned in Sect. 4.1, after the cylinder is shaken, the suspension of particles is homogeneous. Then, these particles start sedimenting under the action of gravity and interacts with the flow under drag and squeezing forces, see Eq. (7) and Eq. (10). Prior to the beginning of the simulation, a homogeneous distribution of particles has to be defined as initial conditions for the numerical modelling.

The samples are composed of a set of particles, whose sizes follow a specific distribution. The set is defined by: i) several different diameters $\phi_{n}$ with $n$ ranging from 1 to $n_{\phi_{n}}=220$, and ii) the number of particles $\left.n\right|_{\phi_{n}}$ associated to each $\phi_{n}$. 


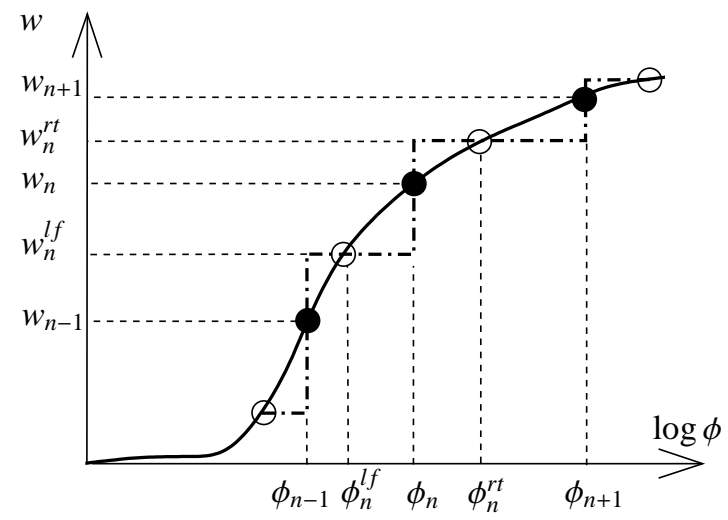

Figure 5: Continuous (thick) and discretized piecewise (dotted line) grading size distribution: accumulated weight $w$ in \% vs. particle diameters.

Figure 5 schematizes the granulometry; the abscissa represents the diameter $\phi$ and the ordinate the percentage of accumulated weight $w$ (weight of particles with diameters less than or equal to a given one defined by a subindex). The figure plots with a thick line the particle size distribution of a generic sample. Since the real distribution is continuous but DEM must manage a finite number of sizes, the curve is discretized into $n_{\phi_{n}}$ points represented by the solid black bullets. The weight above the diameter $\phi_{n}$ is approximated by the average of $w_{n}^{\text {lf }}$ corresponding to $\phi_{n}^{l f}=\left(\phi_{n-1}+\phi_{n}\right) / 2$ and $w_{n}^{r t}$ corresponding to a $\phi_{n}^{r t}=\left(\phi_{n}+\phi_{n+1}\right) / 2$ from the original distribution. These left and right diameters are represented in the curve by hollow circles. Given a sample of mass $m_{t t}$ and a set of $\phi_{n}$, the number of particles corresponding to each diameter is obtained as

$$
\left.n\right|_{\phi_{n}}=6 m_{t t} \frac{w_{n}^{r t}-w_{n}^{l f}}{100 \rho_{p} \pi \phi_{n}^{3}},
$$

where $w_{n}^{l f}, w_{n}^{r t}$ are the accumulated weights (in percent) of the left and right diameters around the midpoint. Therefore, the original distribution is replaced by the discrete (dotted line) defined by the relations $\phi_{n} \leftrightarrow w_{n}$. The piecewise distribution tends to the original one as $n_{\phi_{n}}$ increases.

In the laboratory test, a total of $n_{t t}=\sum_{n=1}^{n_{\phi_{n}}} n_{p \phi_{n}}$ particles representing the $60 \times 10^{-3} \mathrm{Kg}$ of the sample is poured and shaken in a standard cylinder of height $H=0.457 \mathrm{~m}$ and diameter $B=0.0635 \mathrm{~m}$ with a geometrical ratio $r_{c y}=B / H$ $=0.14$.

The particle motion in the three tests is approximately vertical and axisymmetric due to the small size and the negligible horizontal post-collision velocities of the particles. Therefore, a $2 \mathrm{D}$ rectangular model of the cylinder vertical section is used for simulation, reducing the CPU cost since a much lower number of particles is required, without compromising the reproduction of the physics of sedimentation. We choose to model the equivalent of two opposing wedges of $1^{\circ}$, that is, $180^{\text {th }}$ of the total volume; these two wedges are projected onto a $2 \mathrm{D}$ rectangle of width $B$ and height $H$. The rectangle is divided onto a regular $2 \mathrm{D}$ ma-

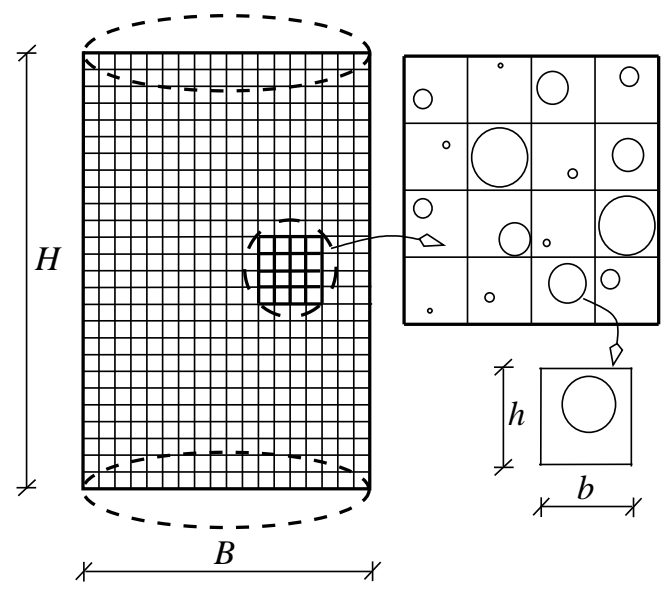

Figure 6: Two-dimensional rectangular matrix: particles randomly located in cells (stratified random sampling). Positions represent the homogeneous suspension after shaking.

trix of $n_{\text {num }} \times n_{\text {num }}$, with $n_{\text {num }}$ equal to $\sqrt{n_{t t} / 180}$ rounded up to the next integer (see Fig. 6 left). The cells have the same aspect ratio as the cylinder. The particles, amounting to a total mass of $60 \mathrm{gr} / 180^{\circ}$, are randomly stratified inside the cells, see Fig. 6 right.

\subsection{Simulation of tests}

As particles settle they are progressively gradated in the cylinder according to its size and therefore particle concentration varies within the cylinder and in time. The one-way coupled DEM computes the positions of the several-million particles by integrating Eqs. (5) at all time steps. At any given time step particle concentrations can be computed for specified volumes at given depths in the cylinder. Next, we describe how the results from DEM are used to mimic the laboratory tests.

\subsubsection{Simulation of the ASTM-D422 hydrometer test}

To measure fluid density (or equivalently particle concentration) at different instants, an ASTM-E100 hydrometer model 152- $\mathrm{H}$ (dimensions in Fig. 7) is sunken in a standardized cylinder full of water with a particle suspension. This type of hydrometer is suitable for measuring the range of densities expected in the simulation. Although it would have been possible to include the hydrometer itself as another particle in the numerical model, it would have required specific formulation for its equilibrium equation and would increase the CPU time as explained in [39]. We decide not to include it in the simulation since its presence does not affect the particles' sedimentation. (Including the hydrometer in the simulation could help to optimize its shape, weight, etc.)

Figure 8 depicts the physical functioning of the hydrometer. In the real experiment, depicted in the left side, the hydrometer would sink to a depth $y_{o}$ at certain $t_{o}$. With these two values a limiting diameter $\phi_{o}$ of all particles 


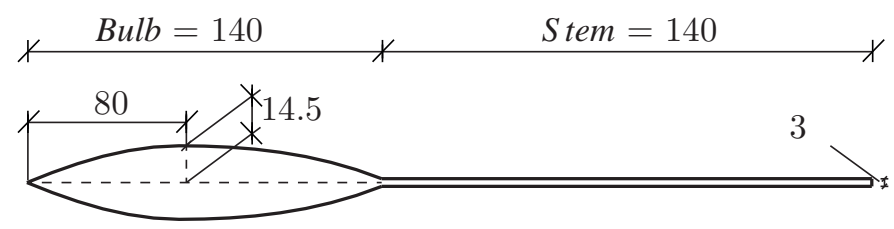

Figure 7: ASTM-E100 hydrometer model 152-H, dimensions in mm.

above $y_{o}$ is determined by the following expression:

$$
\phi_{o}=\sqrt{\frac{18 v_{f}}{\left(\rho_{p}-\rho_{f}\right)|\boldsymbol{g}|}} \sqrt{\frac{y_{g o}}{t_{o}}} .
$$

(An expression very similar to that in (3).) The accumulated weight for all particles with diameters below this limited diameter $\phi_{o}$ is given by

$$
w_{o}=100 \frac{c_{o}}{c_{0}} .
$$

with $c_{0}$ the particle concentration at time zero, which is known, and $c_{o}$ the particle concentration at time $t_{o}$, which is given by

$$
c_{o}=\frac{\rho_{p}\left(\rho_{s}-\rho_{f}\right)}{\rho_{p}-\rho_{f}}
$$

where $\rho_{s}$ is the suspension density, which is read at the hydrometer stem as a function of $y_{o}$.

Combining the previous expressions, the accumulated weight equation of the projected parallelepiped is obtained by

$$
w_{o}=100 \frac{\rho_{p} \rho_{f}}{\rho_{p}-\rho_{f}} \frac{v_{0}}{m_{t t}}\left(r_{s}-1\right)
$$

where $r_{s}=\rho_{s} / \rho_{f}$, given by $[40]$

$$
r_{s}=\frac{m_{t t}}{m_{t t}-A\left(y_{o w}-y_{o}\right) \rho_{f}},
$$

where $A$ is the cross-sectional area of the hydrometer stem and $y_{o w}$ the depth at which the same hydrometer would sink in pure water.

As a summary, to obtain the diameter $\phi o$ and the associated accumulated weight $w_{o}$ it is necessary to measure, in the laboratory, the depth of the center of gravity of the bulb and the density of the suspension around the bulb.

The standard prescribes that depths $y_{0}$ must be measured at ordered instants $t_{o}=\{2,5,15,30,60,250,1440\}$ min, and the resulting pairs $\left(\phi_{o}, w_{o}\right)$ obtained after equations (16) and (17) the granulometric curve is established.

The numerical simulation of the experiment proceeds first by computing the depth at which the hydrometer would sink, establishing the equilibrium of the hydrometer weight $\boldsymbol{w}_{h d}$ and the resultant of hydrostatic forces $\boldsymbol{f}_{h}^{j}$ as schematized in Fig 8 right. The density of the suspension, and therefore the pressure exerted on the hydrometer, is not constant as explained in [35]. For this reason, the computation of the $y_{o}$ is not straightforward; it is computed from the positions of the particles as described next

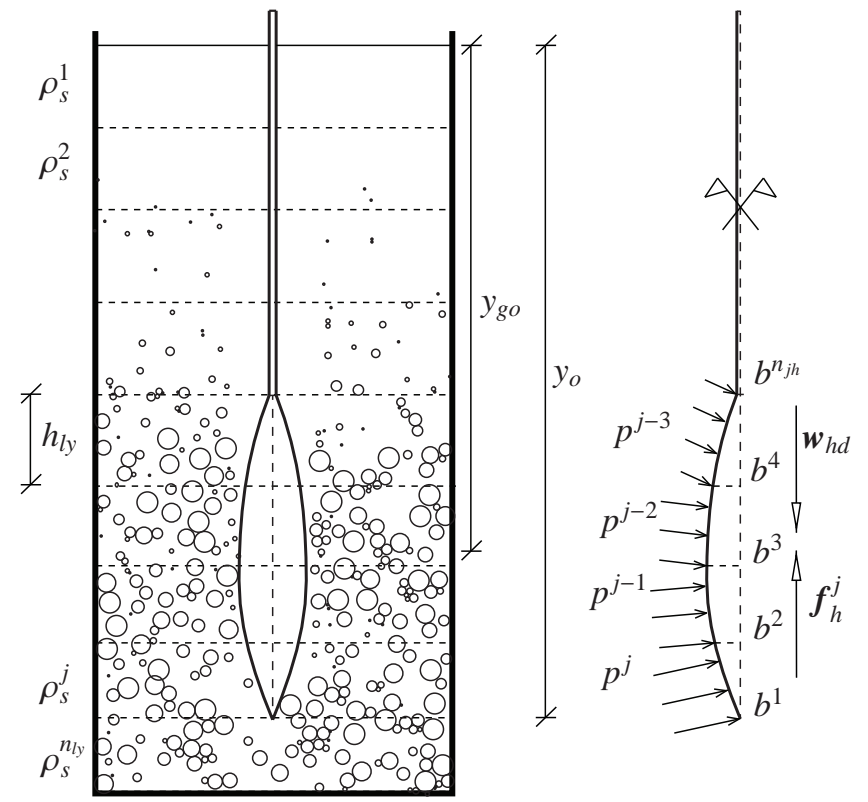

Figure 8: ASTM D422 test: hydrometer sunken in suspension with particles artificially enlarged. Distribution of densities (left); pressures and force equilibrium (right).

- Discretization of $H$ in $n_{l y}$ equally spaced layers of height $h_{l y}$, see Fig. 8 left.

Each layer must contain a statistically representative number of particles; by trial and error we choose $n_{l y}=$ 4000 with a resulting $h_{l y}=H / n_{l y}=115 \times 10^{-6} \mathrm{~m}$, that is, each layer is as thick as about 46 particles of the minimum diameter and as about 1.5 particles of the maximum per layer. For the purpose of computing solution densities, it is necessary to assign a thickness $h_{z}$ to the $2 \mathrm{D}$ projection of the two 10 wedges, this thickness must be such that the total volume of the resulting parallelepiped coincides with that of the two wedges, which results in $h_{z}=\pi B / 720=2.26 \times 10^{-4} \mathrm{~m}$.

- For layer $j\left(j=1, \ldots, n_{l y}\right.$, counting down from the liquid free surface), the density of the suspension $\rho_{s}^{j}$ is given by

$$
\rho_{s}^{j}=\frac{\rho_{p} v_{p}^{j}+\rho_{f} v_{f}^{j}}{v_{t t}^{j}},
$$

where $v_{t t}^{j}=B h_{l y} h_{z}$ is the total volume of the layer, and $v_{p}^{j}=v_{t t}^{j}-v_{f}^{j}$ is the volume of the particles in the layer, which is calculated using DEM. The density of the suspensions only has to be calculated for the layers that comprise the bulb.

- The vertical hydrostatic force is the resultant of the pressures acting on the hydrometer bulb.

For a layer $j$, the pressure is the weight of a unit-area column from the bottom point of the layer to the liquid 
free surface, which is computed by adding up the weights of all layers above

$$
p^{j}=\sum_{n=1}^{j} \frac{\rho_{s}^{n} v_{c o}^{n}|\boldsymbol{g}|}{B h_{z}}
$$

where $v_{c o}^{n}$ is the volume of layer $n$. This pressure is a vector orthogonal to the bulb surface.

The hydrostatic force acting on the bulb is the summation of the pressures exerted by each layer on the horizontal projection of the intersection of the layer with the hydrometer bulb. Therefore, for a hydrometer with its tip immersed in $j$, and with its bulb intersecting $n_{j h}$ layers the total hydrostatic force is

$$
\boldsymbol{f}_{h}^{j}=\sum_{n=1}^{n_{j h}-1}\left(p^{j-n+1} \cdot \boldsymbol{j}\right) \pi\left[\left(b^{n+1}\right)^{2}-\left(b^{n}\right)^{2}\right],
$$

where $\boldsymbol{j}$ is the unit vertical vector, the dot - represents the scalar product and $b^{n}$ the bulb radii at the intersection with the layer boundaries.

- In equilibrium, the tip of the hydrometer sinks to $y_{o}$ (located inside a layer $j$ ), and the hydrometer weight $\boldsymbol{w}_{h d}$ balances $\boldsymbol{f}_{h}^{j}$.

The equilibrium depth is found iteratively varying the position of the bulb tip until $\left|\boldsymbol{f}_{h}^{j-1}\right|<\left|\boldsymbol{w}_{h d}\right|<\left|\boldsymbol{f}_{h}^{j}\right|$, corresponding to a depth $y_{o}$ between $(j-1) h_{l y}$ and $j h_{l y}$. If the number of layers is large (as is the case of this experiment), that is, the thickness of the layers is small, we can assume that the tip of the hydrometer is at the bottom of the layer, but if the layer thicknesses are large it is best to assume a varying pressure within the layer and linearly interpolate the position of the tip as

$$
y_{o}=j h_{l y}+\frac{j h_{l y}-(j-1) h_{l y}}{\left|\boldsymbol{f}_{h}^{j}\right|-\left|\boldsymbol{f}_{h}^{j-1}\right|}\left(\left|\boldsymbol{w}_{h d}\right|-\left|\boldsymbol{f}_{h}^{j-1}\right|\right) .
$$

Once the depth of the tip of the hydrometer is known, the depth of its center of gravity is computed, and the density of the solution is obtained using (20) considering a single layer extending the height of the bulb. From these two parameters, the pairs $\left(\phi_{o}, w_{o}\right)$ are obtained as in the standard.

During the DEM simulation, particle positions are stored every $10 \mathrm{~s}$. Therefore we could simulate the position of the bulb, and the suspension density at any instant after the initial shaking.

\subsubsection{Simulation of the buoyancy test}

In this variation of the previous test, a precision dynamometer (Fig. 9 left) measures the tensile force $\boldsymbol{f}_{b t}$ acting on the line, which must be equal to $\boldsymbol{w}_{s p}-\boldsymbol{f}_{h}$, where $\boldsymbol{w}_{s p}$ is the sphere weight, and $\boldsymbol{f}_{h}=-v_{s p} \rho_{s} \boldsymbol{g}$ is the (time varying) hydrostatic force at the depth of the sphere $y_{b t}$, where $v_{s p}$ is the volume of the sphere and $\rho_{s}$ is the suspension density at that depth. The apparent mass of the sphere is therefore $m_{s p}=\left|\boldsymbol{f}_{b t}\right| /|\boldsymbol{g}|$. The suspension density is numerically computed by DEM following the same procedure described for the previous test and Eq. (20). Again, to reduce the CPU cost, the sphere is not simulated as an additional particle for the same reasons as we did not simulate the hydrometer in the ASTM test.

The apparent mass $m_{s p}\left(t_{o}\right)$ is measured in this test at the ordered instants $t_{o}=\{0.5,1,2,4,15,30,60,120,240,480\}$ min. At each time, a diameter $\phi_{o}$ is determined with equation (16) but replacing $y_{g o}$ by $y_{b t}$, which remains constant through the experiment. And the percentage of accumulated weight of particles with diameter $\leq \phi_{o}$ is given by

$$
w_{o}=100 \frac{\rho_{f}}{c_{0}} \frac{\left.m_{s p}\right|_{w}-m_{s p}}{\left(\frac{\rho_{f}}{\rho_{p}}-1\right)\left(\left.m_{s p}\right|_{w}-\left.m_{s p}\right|_{a}\right)},
$$

where $\left.m_{s p}\right|_{w}$ is the apparent mass that the sphere would have if immersed in pure water to the same depth, and $\left.m_{s p}\right|_{a}$ is the mass of the sphere in air.

In the numerical simulation, we determine the apparent mass of the sphere by subtracting from the mass of the sphere, the mass of the solution which occupies the same volume as the sphere.
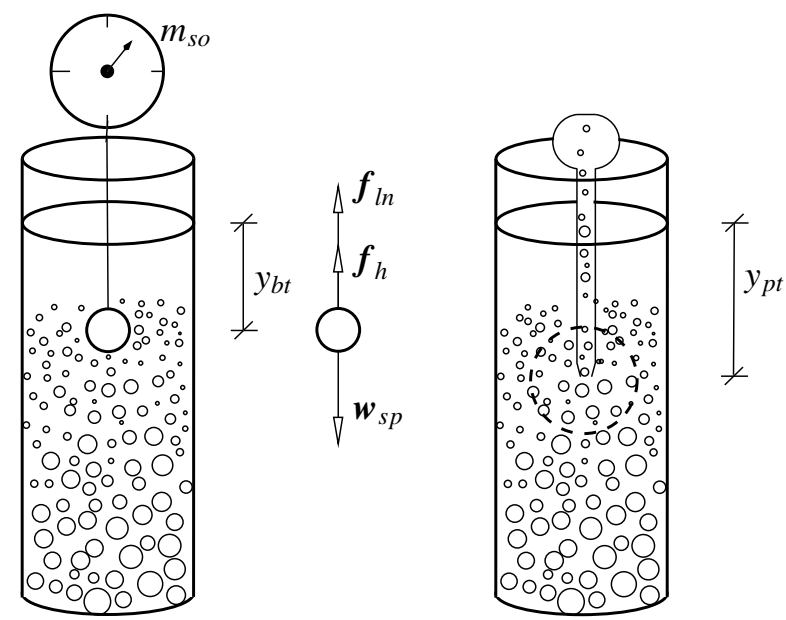

Figure 9: Buoyancy test (left): force equilibrium and scale measuring apparent mass $m_{s p}$ of an immersed sphere at constant depth $y_{b t}$. Pipette test (right): extraction of $35 \mathrm{ml}$ suspension subsample at constant $y_{p t}$ around circle. Particles artificially enlarged for clarity.

\subsubsection{Simulation of the pipette test}

This test measures the concentration $c_{o}$ of the suspension at a fixed depth $y_{p t}$ and at the axis of symmetry of the cylinder for several ordered instants $t_{o}$ by extracting a small amount of suspension with a standard pipette, see Fig. 9 right; we have not found in the literature any data of the times that should be used for the sampling. The subsample is dried and the mass $m_{p t}$ weighted; then, the 
concentration is directly calculated dividing this weight by the subsample volume.

The accumulated weight is calculated with the same expressions as those of ASTM-D422 with $y_{g o} \equiv y_{p t}$. Again, a set of data pairs $\left(\phi_{o}, w_{o}\right)$ provides the particle size distribution as in Fig. 5.

In the numerical simulation, The composition of the suspension is calculated from the simulation of DEM by sampling the particles' position and diameters inside a circle centered at the vertical centerline of the cylinder and at the prescribed position of the pipette tip $y_{p t}$, see Fig. 9 right. The circle radius is chosen so that the sampling volume is equivalent to that of the experiment accounting for the fact that we simulate only two wedges of one $\circ$ of the whole cylinder.

\subsection{Numerical solution}

The procedure to obtain the numerical solution starts with the input of material, numerical parameters and the initial location of particles described in Sect. 4.4. The initial velocity is set to zero; the computer code simulates the sinking through changes of positions and velocities for all particles taking into account gravity $\boldsymbol{w}^{i}, \operatorname{drag} \boldsymbol{f}_{d}^{i}$, contact $\boldsymbol{f}_{c}^{i}$, lubrication $\boldsymbol{f}_{l}^{i}$ and hydrostatic $\boldsymbol{f}_{h}^{i}$ forces acting on all particles.

DEM detects contacts applying Eq. (1) to all particles to compute the corresponding contact $\boldsymbol{f}_{c}^{i k}$ and lubrication $\boldsymbol{f}_{l}^{i k}$ forces. Traditionally this contacts are computed for all possible data pairs in order to reduce computational time. In this work, the efficient procedure described by [41] has been used, checking particle contacts only with the closest particles and/or boundaries.

The contact and lubrication forces are used in the governing Eqs. (5), forming a set of non-linear differential equations, which are solved through time with an iterative finite-difference algorithm. We have used a time integration scheme specifically designed for the consistent energy dissipation of contact, drag and lubrication non-linearities, see [42] and [34]. The CPU time is further reduced using a variable time step $\Delta t$, with an upper limit to suppress spurious oscillations in particle velocities. The terminal velocity $\left|\dot{\boldsymbol{u}}_{t r}^{i}\right|$ and its related terminal time $t_{t r}^{i}$ from Sect. 4.3 defines the initial time increment used to integrate the Eqs. (5): $\Delta t$ has to be smaller than the $t_{t r}^{i}$ of the smallest particle. Furthermore, to ensure accuracy besides stability, we use $\Delta t \leq 0.1 \min \left(t_{t r}^{i}\right)$. At certain chosen time step, a computer routine checks that all particles have reached their $\dot{\boldsymbol{u}}_{t r}$; then, the $\Delta t$ from the previous iteration is progressively increased until unstable changes in the velocity appear again, then this $\Delta t$ is reduced a small amount and the check is repeated.

The set of equations is linearized with standard NewtonRaphson iterative techniques.

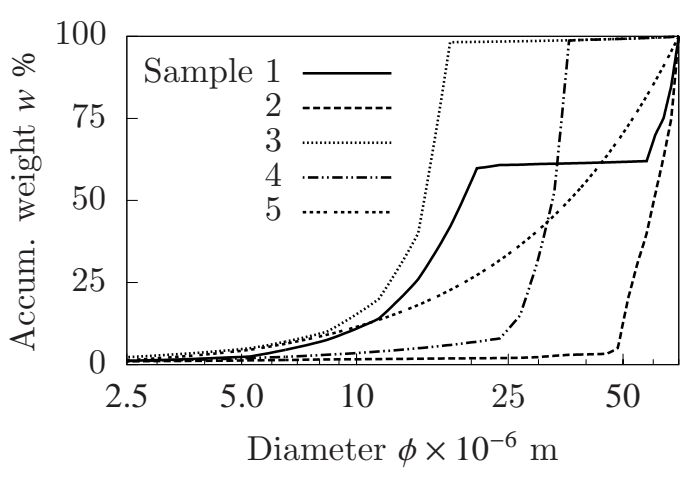

Figure 10: Particle size distribution (granulometry) for five representative samples with diameters between $2.5 \times 10^{-6} \mathrm{~m}$ and $70 \times 10^{-6}$ m.

\section{Numerical sedimentation and particle size dis- tribution}

This section presents the results of the numerical analysis for the particle sedimentation and describes their most important features. Additionally, the simulations provide useful insight into the performance and quality of the three test designs.

\subsection{Initial granulometry}

Five grading size distributions or "samples" have been studied, see Fig. 10. A sample is taken from each distribution, all samples have the same mass, and contain particles with diameters ranging from $2.5 \times 10^{-6} \mathrm{~m}$ to $70 \times 10^{-6} \mathrm{~m}$ and at least one particle for each $\phi_{n}$ in which the grading curve has been discretized; the range of sizes can be seen in Fig. 10. The size distributions have been chosen to be very different among them and to include discontinuities in order to investigate the measurement capabilities of the test methods.

The first sample is composed mostly of very small particles with diameters from $2.5 \times 10^{-6} \mathrm{~m}$ to $20 \times 10^{-6} \mathrm{~m}$ with some large ones of minimum diameter of $60 \times 10^{-6} \mathrm{~m}$; the large particles amount for $40 \%$ of the total weight; two rising limbs and a plateau in between can be observed in the size distribution curve. In the second sample, large diameters from $50 \times 10^{-6} \mathrm{~m}$ to $70 \times 10^{-6} \mathrm{~m}$ are prevalent, comprising $95 \%$ of the weight; the distribution has a single rising limb. In sample 3, small particles from $2.5 \times 10^{-6} \mathrm{~m}$ to $15 \times 10^{-6} \mathrm{~m}$ are dominant, accounting for $95 \%$ of the total weight; the distribution has a single rising limb. In sample $4,92 \%$ is composed by particles with diameters between $25 \times 10^{-6} \mathrm{~m}$ to $35 \times 10^{-6} \mathrm{~m}, 5 \%$ of the sample has smaller diameters, and 3\% larger ones. Finally, sample 5 has a smooth distribution with gradated diameters of approximately equal contribution to the total weight.

Before carrying out the simulation, terminal velocities and the times to reach them are calculated for several representative particle sizes using Eqs. (13). Table 1 shows $\left|\dot{\boldsymbol{u}}_{t r}\right|$ and $t_{t r}$ for single particles sedimenting in water. It can 
be appreciated that both quantities strongly depend on size and that the highest velocity is three orders of magnitude larger than the smallest one.

ASTMD-422 requires that measurements must be taken once all particles have reached terminal velocity and transient phenomena have disappeared, prescribing that the first measurement must be done after the first minute of sedimentation.

\subsection{General simulation}

For all samples, the penalty parameters are $K_{N}=K_{T}=$ $10^{3} \mathrm{~N} / \mathrm{m}$, values based on trial and error to avoid numerical ill conditioning due to the very small size of some particles. As mentioned in Sect. 4.6, the initial $\Delta t$ must be small enough to capture the transient particle motion shown in Fig. 4 ; thus, an initial $\Delta t \approx 2.6 \times 10^{-7} \mathrm{~s}$ is adopted. The other parameters have been given in the previous sections.

In Fig. 11 the numerical sedimentation simulation is shown for 60, 300 and $900 \mathrm{~s}$ (from top to bottom) and for the five samples (from left to right); the long rectangles represent the cylinder. For display purposes, a zoom is made at the top, intermediate and bottom areas of the cylinder, with magnification rates of 25, 25 and 62.5 , respectively, at $900 \mathrm{~s}$; the smallest particles cannot be seen due to limited resolution.

The grey scale in the cylinders represent the mass proportion with respect to the total mass of $60 \mathrm{gr}$, averaged every 100 layers out of the total 4000 in which the cylinder was discretized, see Sect. 4.5.1. Right after the initial shaking, the particles' positions are equally distributed; for this reason, at $60 \mathrm{~s}$ in most of the samples the grey intensity is fairly uniform, except at the top where all intermediate and large particles have sedimented and at the bottom, where some of these particles rest without movement. The exception is for sample 2, in which the prevalence of large particles shows a small or very small concentration in the upper half of the cylinder.

At $300 \mathrm{~s}$ (middle figures), sedimentation is in an intermediate state, with particles distributed within most of the cylinder except in sample 2 for which the sedimentation is nearly finished. The granulometry of the samples starts to be evident, for example in number 5 the gradation is linear and in sample 3 most of the particles still fill the cylinder.

At $900 \mathrm{~s}$ (bottom figures), only small sizes remain at the top and middle of the cylinder; at the bottom there is a mixture of small, intermediate and large particles, the latter the most abundant due to their high deposition velocity. Therefore, and except for sample 3, most of the mass is at the cylinder base as shown by the almost black intensity.

In accordance to the experimental observations from [43], the particles progressively gradate in horizontal layers. This can be visually verified in the first sample and especially in the third one, in which large, intermediate and small particles group in sets due to the similar deposition velocity of similar sizes.
During the simulation, the fluid interacts with the particles, mostly with the large ones due to their higher Reynolds number. The contact interactions are also important, large particles sediment with higher terminal velocity $\left|\dot{\boldsymbol{u}}_{t r}\right|$ (Fig. 4) and, therefore, overtake the smaller ones. In this process, clusters of particles with different sizes but with almost the same high velocity may form: small particles are "trapped" by the large ones. Some clusters can be appreciated in the middle zooms of samples 1 and 5 but, we have concluded that, for the sedimentation analyses considered here, there is no need for a special treatment of the clusters since they occur towards the lower part of the cylinder, away from the area in which the experimental measurements are modeled.

Although the CPU time of each simulation is reduced using incrementally larger time steps and restricted contact search (see Sect. 4.6), this time is still very high when the whole process is simulated. For sample 3, the simulation takes about 420 hours in an Intel (R)station Core i7-4930K, with 12 cores running at $3.40 \mathrm{GHz}$ and with 32 $\mathrm{Gb}$ of RAM memory. This is due to the large number of small particles and time steps. The fastest simulation occurs for sample 2, the one with the smallest number of particles, which takes 60 hours.

Every $10^{-4} \mathrm{~s}$ the positions and velocities of all particles are recorded, and they are used, together with the particle diameters, to compute the intermediate data, densities, concentrations, depths and apparent masses needed to apply the analytical laws from Sects. 4.5.1, 4.5.2 and 4.5.3 to infer the particle size distributions as if the experiments had been carried out.

\subsection{Preliminary measurements}

Figure 12 shows, for different times, the limiting diameter of all particles above depth $y_{c y}$ as given by Eq. 16 together with the minimum diameter of the particles actually above $y_{c y}$ as modeled numerically. Depth varies from the free surface $y_{c y}=0$ to the bottom of the cylinder $y_{c y}=H=0.457 \mathrm{~m}$, (geometry in Fig. 6) and the times displayed are 60 s, 120 s, 300 s, and 900 s. Since the samples have the same maximum diameter the curves will coincide for the five of them.

The agreement between the values given by Eq. (16) and the maximum diameter observed in the numerical experiments is remarkable, with the following exceptions. At $60 \mathrm{~s}$, the numerical values are below the analytical results, but this is consistent with the fact that Eq. (16) does not pretend to give the exact value but an upper bound (see Sect. 4.5), and, therefore, it can overestimate the actual value. For instance, this equation predicts that at a depth of $0.4 \mathrm{~m}$, the limiting diameter is $90 \times 10^{-6} \mathrm{~m}$, but we do not have any sample with such a diameter, so the numerical results are bounded by the maximum diameter in the sample.

Finally, at $300 \mathrm{~s}$ and at $900 \mathrm{~s}$, the agreement is good for the upper part of the cylinder, but it fails at the bottom, because sediment accumulate and Eq. (16) is for a 

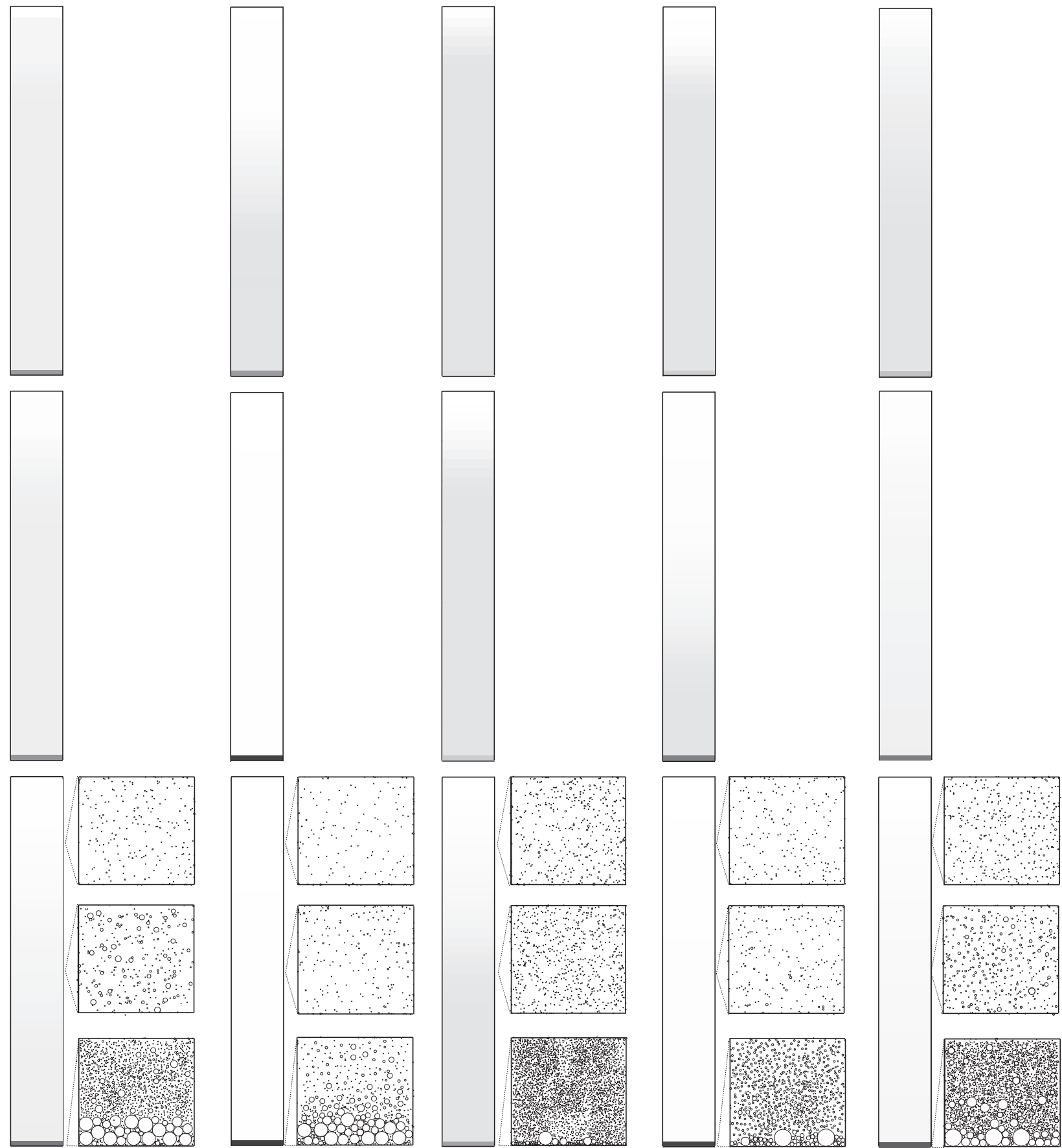

Figure 11: Numerical simulation of the ASTM-D422 experiment for the five samples. Gradation of particles at 60, 300 and $900 \mathrm{~s}$. The grey scale represents the proportion of mass with respect to the total mass. Zooms of particles with amplification of 25 (top and middle) and 62.5 (bottom). 
bottomless cylinder, and it does not account for a possible accumulation of sediments.

For later times the three curves become flatter since only very small particles would remain in suspension. They also would tend to collapse to the minimum diameter except close to the bottom zone, in which the numerical method "knows" that all particles are accumulating there.

Figure 13 shows the variability of the suspension density along the height of the bulb, from top to bottom $0 \leq h_{h d} \leq 0.14$ (see Figs. 7 and 8 for geometry), for the different samples and at different times. The average suspension density along the height of the bulb is the key variable in Eq. (18) to determine the weight percentage of the particles above the limiting diameter associated to the bulb-center depth. Also notice that since the bulb sinks as the experiment progresses, the curves correspond to different depths in the sedimentation cylinder. The curves at time $t=0$ are approximately horizontal and equal for all samples corresponding to the density of a homogeneous suspension of density $1015 \mathrm{~kg} / \mathrm{m}^{3}$. For $t=900 \mathrm{~s}$, the curves are again almost horizontal and with densities getting closer to the fluid density, samples 1, 3 and 5, which contain a larger proportion of fine material than the other two samples have a density higher than the fluid density since the finest particles are still in suspension. For the intermediate times, we can observe different density distributions: for sample 1, the coincidence of the curves at times $60 \mathrm{~s}$ and $180 \mathrm{~s}$ is associated to the discontinuity in the particle size distribution; for sample 2 , at time $60 \mathrm{~s}$, there is an approximately linear variability along the bulb, all particles have already passed the upper part of the bulb, and a few of the smaller ones are still sedimenting around the bulb center and below; for sample 3, the coincidence of the curves at times 0 and $60 \mathrm{~s}$ is due to the small diameter of the larger particles in the sample, at time $60 \mathrm{~s}$, at the depth of the bulb there has not been a differentiation of the particles, at times $180 \mathrm{~s}$ and $300 \mathrm{~s}$ it is when a linear variability of the density can be observed, corresponding to a moment in which the small and intermediate particles are passing by the bulb; for sample 4, the behavior is exactly the same as for sample 3, at the beginning, all particles are too small and have not displayed their differential velocities at the bulb depth, sometime between $60 \mathrm{~s}$ and $180 \mathrm{~s}$, the suspension should have displayed a linear variation similar to the one observed for sample 3, but which was not captured given the larger diameters of the particles for this sample; for sample 5, it is at time 60 $\mathrm{s}$ when the linear variability of the suspension density is observed, after that the density diminishes with time in an almost constant distribution along the bulb.

Comparing the average densities about the bulb computed by averaging the curves in Fig. 13 and those obtained by the approximate expression given by Eq. (19), their values are almost exactly equal except for samples 3 and 4, for which there are some deviations as shown in Fig. 14. The differences are due to the fact that the approximate expression assumes a homogenous suspen- sion density, whereas, as discussed above, in the curves in Fig. 13 the suspension density varies approximately linearly for some time steps.

\subsection{Tests measurements}

This subsection presents relevant intermediate results for the three tests described in Sect. 4.5. In Fig. 15 the hydrometer depth $y_{g o}$, limiting diameter $\phi_{o}$ and accumulated weight percentage $w_{o}$ computed after the numerical simulation of the ASTM hydrometer test are shown for the five samples. Through the duration of the test, the suspension density $\rho_{s}$ decreases at the top and middle of the cylinder and increases at the bottom, with the corresponding spatial variation of pressure and hydrostatic force (see Fig. 8) on the bulb. Due to the progressive decrease of $\rho_{s}$ around the bulb, the hydrometer sinks with time ( $y_{g o}$ increases), since to reach equilibrium, a higher depth is needed to balance the weight with the decreasing hydrostatic force. The slope of the curves in the top graph defines the sinking velocity.

Samples 2 and 3 show the highest and lowest sinking velocities at early times, due to their respective preeminence of largest and smallest particle diameters. For sample 1 the slope is zero in the interval 80 to $400 \mathrm{~s}$, for its lack of intermediate particles; during this time interval there is short stationary situation in which similar concentrations of small and large particles are traveling by the bulb. The curve of sample 4 is similar to that of 2 and lies between those of samples 2 and 3 . Finally, for sample number 5 , the hydrometer sinks progressively, due to its particle size gradation. After a sufficient time, $y_{g o}$ asymptotically tends to $y_{w}$, the depth at which the hydrometer would sink in clear water, meaning that most of the particles are concentrated at the cylinder bottom, with the solution density about the bulb approximately equal to that of water, $\rho_{s} \approx \rho_{f}$. When $y_{w}$ is reached, quickly for sample 2 and slowly for 3 , the hydrometer stops sinking.

The progressiveness of sedimentations is also observed in Fig. 15, middle. As particles sink, the large ones rapidly overpass the center of gravity of the bulb, $y_{g o}$ due to their high deposition velocity; this fact can be appreciated with the high slope of the curves at the initial instants, up to $100 \mathrm{~s}$. The values of $\phi_{o}$ are calculated with Eq. (16); at any given time, the resulting limiting diameters are different for the different samples since they vary according to the square root of $y_{g o}$. Again all curves are delimited by those of samples 2 and 3 with the largest and smallest sizes, respectively. Although out of scale in the figure, the asymptotic value slowly approaches the minimum diameter.

The curves in Fig. 15, bottom, show the opposite variation from that observed in the top one. Initially, the concentration is homogeneous and no particle has sedimented and the accumulated weight is the total one. As time passes, the concentration decreases above $y_{g o}$, very fast for samples 2 and 4 and slowly for 1 and 3; therefore the weight percentage of particles that are in suspension 

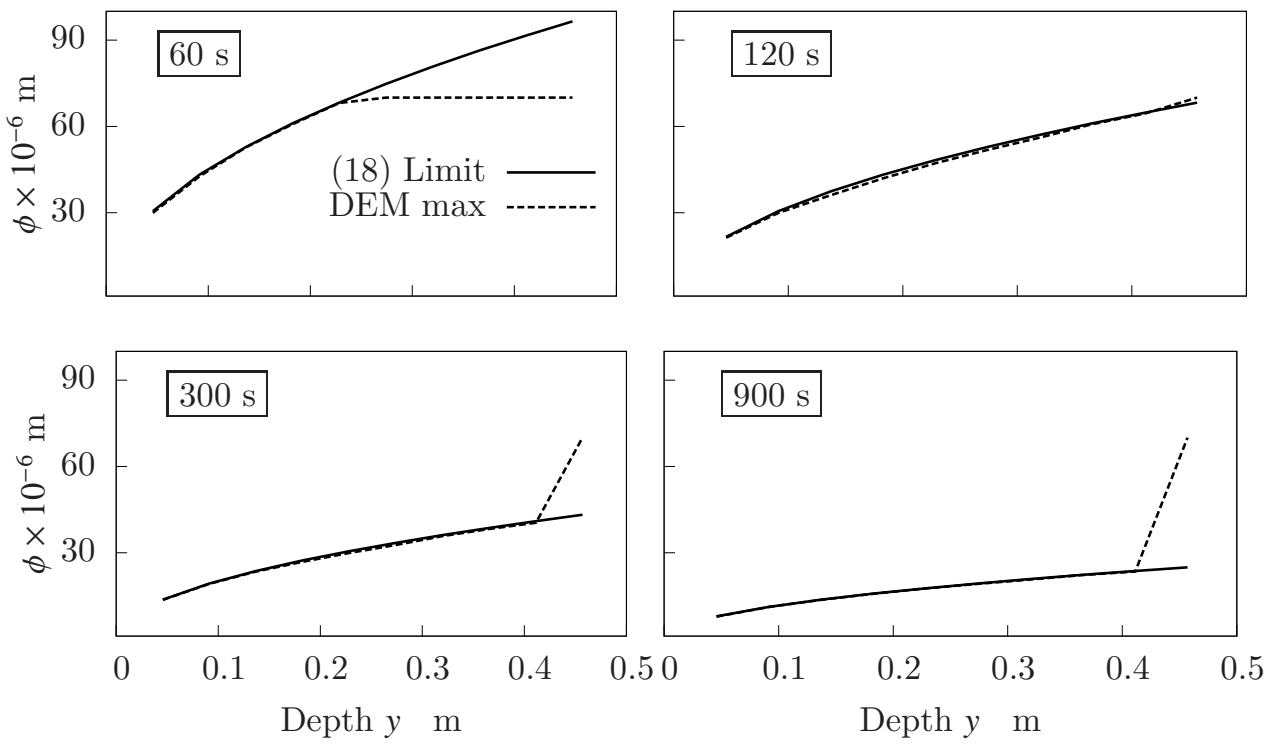

Figure 12: Maximum diameter sizes above a variable depth inside the ASTM cylinder for the five numerical experiments (dashed lines) and limiting diameter from Eq. (16) (continuous line) at different times.
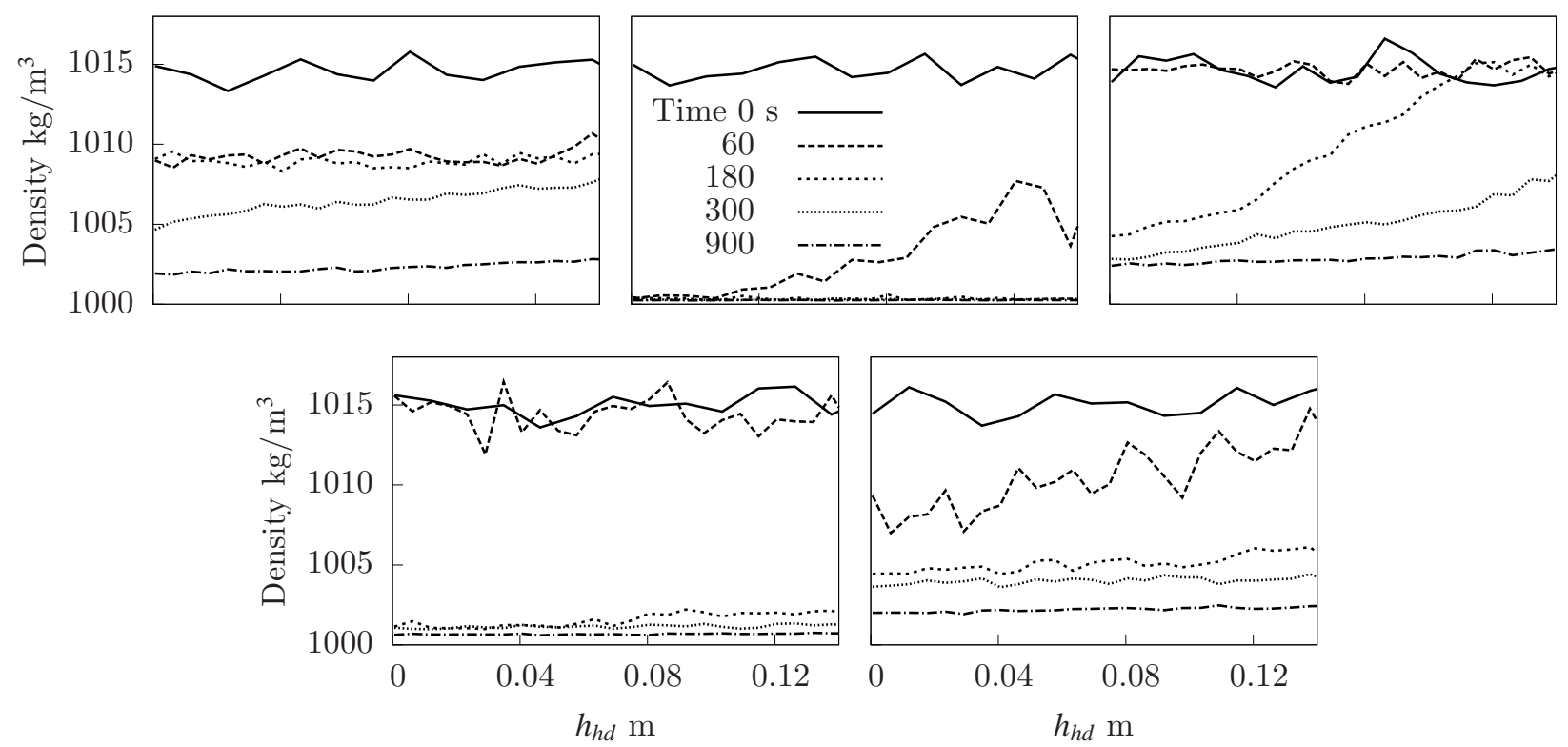

Figure 13: Suspension density $\rho_{s}$ along hydrometer bulb at several instants and for the five samples ordered from left to right, top to bottom.
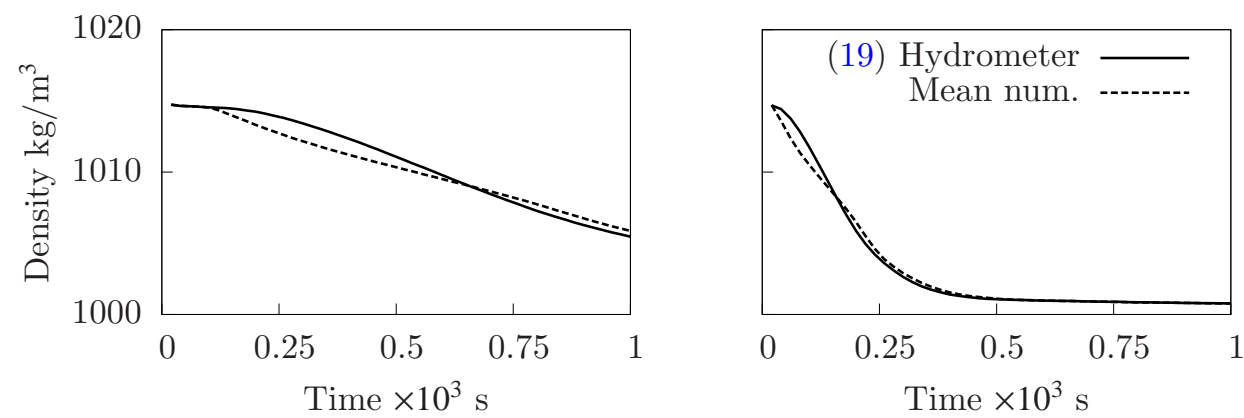

Figure 14: Average suspension density $\rho_{s}$ along hydrometer bulb computed using Eq. (19) (continuous line) and density obtained from numerical simulation, for samples 3 and 4 . 

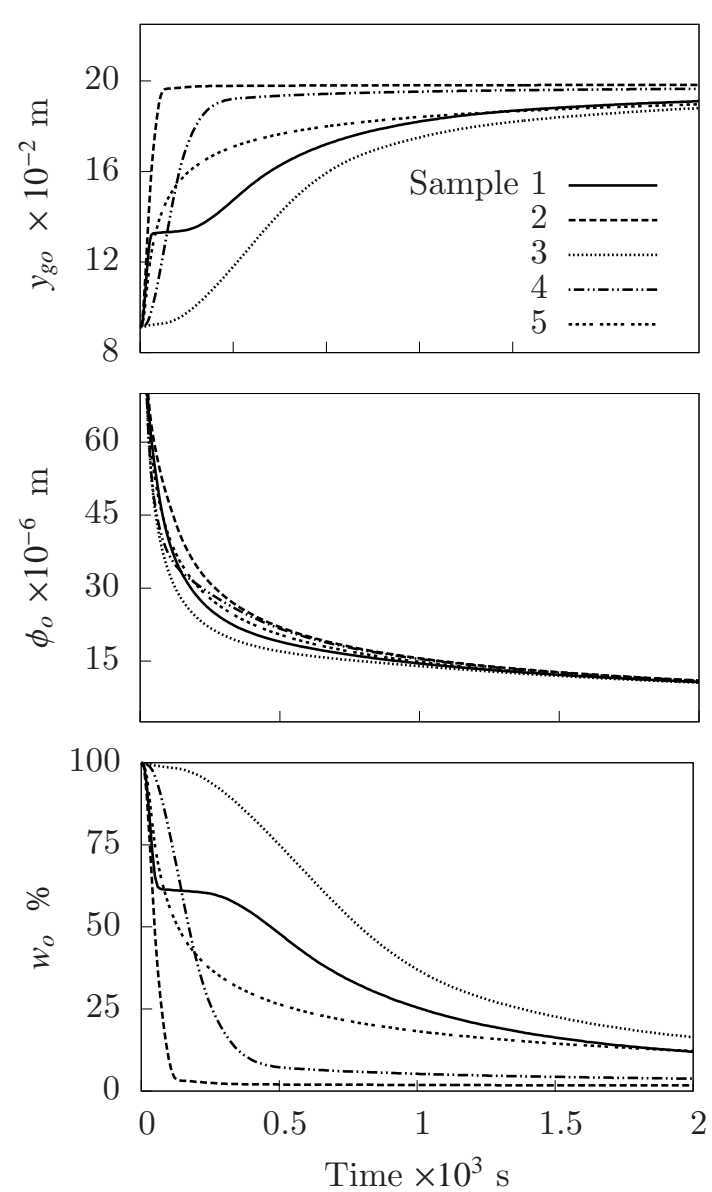

Figure 15: Depth of hydrometer center of gravity (top), limiting diameters (middle) and accumulated weight (bottom) from the simulation of the ASTM-D422 test.

decreases (see Eq. (17)). The value of $w_{o}$ asymptotically tends to zero with time since all particles sediment pass the hydrometer. Again in sample 1 and now in 3 there is a zone of zero slope: a negligible amount of mass passes the bulb in this interval.

Figure 16 shows the apparent mass $m_{s p}$ of the sphere, the limit diameter and the accumulated weight $w_{o}$ vs. time computed for the buoyancy test. The difference between this and the previous tests is that the sphere does not change its position (at $y_{b t}=0.3 H=13.71 \times 10^{-2} \mathrm{~m}$ ) and its weight is balanced by the hydrostatic force and the tension of the line. For reasons discussed in the previous paragraphs, $m_{s p}$ increases when $\rho_{s}$ decreases and, therefore, the hydrostatic force decreases at the depth of the sphere $y_{g o}$ through time.

The top graph in Fig. 16 is similar to the top one in Fig. 15 since both are based in force equilibrium of the same samples, although the response is less smooth in the second. For all samples, the first value is about 1.52 gr, which corresponds to the apparent mass of the sphere submerged in a completely homogeneous suspension, and tends to $1.62 \mathrm{gr}$, which is the apparent mass of the sphere in clear water. The limiting particle diameters $\phi_{o}$ as given
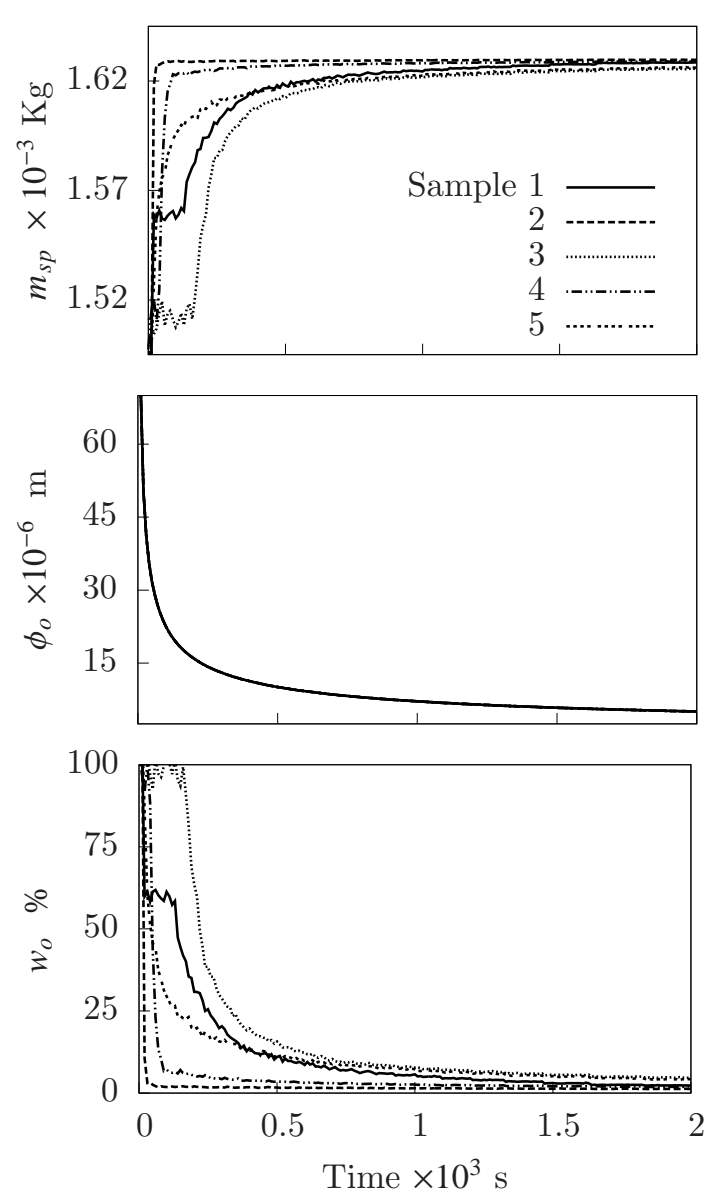

Figure 16: Apparent mass (top), diameters (middle) and accumulated weight (bottom) computed from simulation of the buoyancy test at a depth of $0.3 H$ (see Figs. 6,9 ).

by Eq. (16) reduce to a single value for all samples and a given time since the depth is fixed. The bottom graph in Fig. 16 is also very similar to the corresponding one of Fig. 15 although the decrease in accumulated weight is, in this case, faster for all samples, since the depth of the measurement device is smaller than that of the hydrometer, see again Eq. (16).

The first and last graphs in Fig. 16 show oscillations, specially the top one since the method measures the density of a small portion of suspension close to the sphere, a volume much smaller than that of the hydrometer. In the top graph these oscillations are very visible for sample 3 since the effect of the small measurement area couples with the small diameter of most particles.

Finally, Fig. 17 shows limiting diameters (top) and accumulated weights (bottom) computed with the pipette test at a constant depth equal to the sphere depth in the previous experiment $y_{p t}=y_{b t}$. Both variables follow the same tendency as that of the buoyancy test. The bottom figure is almost equal to that of Fig. 16 although the oscillations are more pronounced, due to the even more reduced sampling volume.

Although not quantified in this work, the higher the 
measurement point of buoyancy and pipette the better and faster the obtained values are, since the gradation is faster and the inhomogeneities also disappear faster.
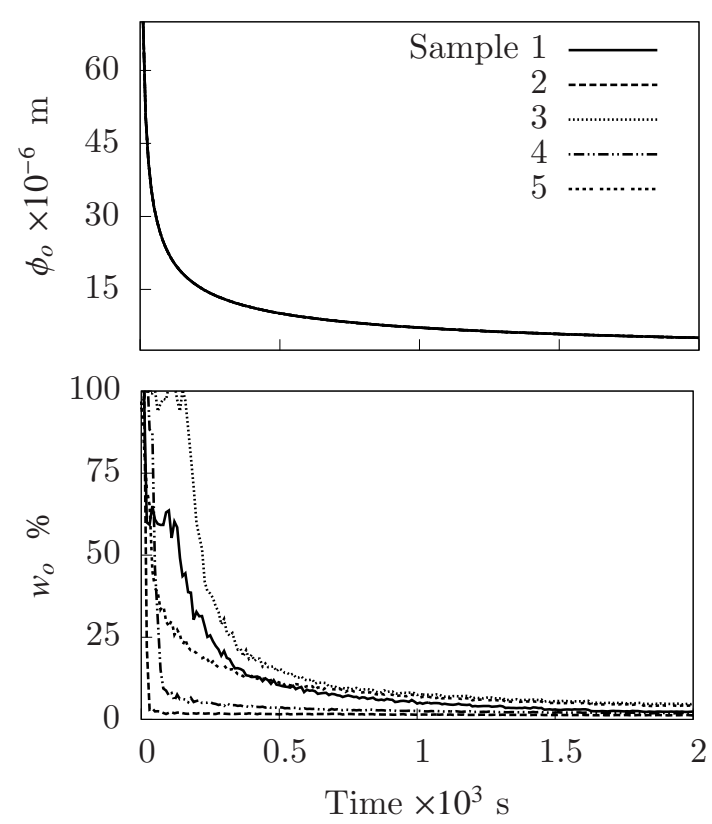

Figure 17: Diameters (top) and accumulated weight computed (bottom) from simulation of the pipette test at a depth of $0.3 H$ (see Figs. 6, 9).

\subsection{Final granulometry}

Figs. 18 compare the original particle size distributions with those numerically calculated, for the three tests and the five samples. These figures are not a validation of the numerical approach but rather a study of the precision of the laboratory tests, since they are based on approximations of some of the physical laws governing the sedimentation process of a group of particle as explained before.

The agreement between the numerically simulated distributions and the original one is very good for small and medium particle sizes, but differences can be found for the large particles since they are measured mostly at the beginning of the experiment when the suspension is composed of a homogeneously mixture of particles of all sizes. This mismatch is an intrinsic shortcoming of the design of these laboratory tests.

The hydrometer test "smoothes" the granulometry and is not capable of capturing discontinuities or the abrupt end of the curve, whereas the buoyancy and pipette tests oscillates for large sizes. This oscillation is due to small and local inhomogeneities at the initial stages of the experiment: even if the particles are supposed to be homogeneously distributed these unavoidable inhomogeneities are relevant in tests that measure locally. The effect is noticeable for samples 4 and 5 that include a fair amount of intermediate particles. The hydrometer test does not have oscillation problems for any sample due to the relatively large volume that it measures, but in exchange tends to predict smaller sizes and slightly lower weights.

The intrinsic inhomogeneities at the beginning are also the cause of the incorrect prediction of accumulated weights above $100 \%$ for the buoyancy and pipette tests: in the relation (17) introduced in Sect. 4.5.1, the local $c_{o}$, at the early stages, can be slightly larger than the average initial $c_{0}$. Note that for all tests, we can compute the weight associated with particle diameters larger than $70 \times 10^{-6} \mathrm{~m}$ (in a real situation we do not know which is the largest particle size), resulting always in percentages of $100 \%$ except for the already mentioned fluctuations.

\section{Conclusions}

The Discrete Element Method (DEM) is a powerful tool for the numerical modelling of systems of large number of particles. Therefore, its application to the numerical simulation of the sedimentation of granular materials is natural and immediate using a "one-way coupling" between fluid and particles. In this work, DEM has permitted to mimic the determination of the particle size distribution of several granular samples by the hydrometer, buoyancy and pipette tests using numerical data and analytical laws of hydrostatics.

The simulation uses the position and velocities of all particles and the description of the real experiments. The particle size distributions using the three tests have been compared with the original ones obtaining a good agreement and most importantly, identifying the strong and weak points in the design of the tests. The detected errors of the real experiment cannot easily be identified with the experimental data. The numerical experiment could be used to optimize the setup and monitor the sedimentation laboratory test in the future with the aim of defining the duration of the experiment, the shape and size of the recipient, or the mass of the sample. We can also envision that this simulation approach could be used to retrieve the exact distribution of the sample from the approximate one obtained in the laboratory by an iterative process based on inverse modelling.

It is worth to emphasize that this work does not intend to supersede the laboratory experiments but permits to understand better their strong and weak points.

The important conclusions from the numerical simulations are:

- Inside the range allowed by the standards, small particles are easier to measure than large ones

- The hydrometer test smoothes the real granulometry, the buoyancy, pipette tests give oscillatory predictions

- All tests tend to predict larger particles than in reality, although with an associated small accumulated weight

- In the buoyancy and pipette tests, the higher the position of the measurement point, the more accurate the prediction 

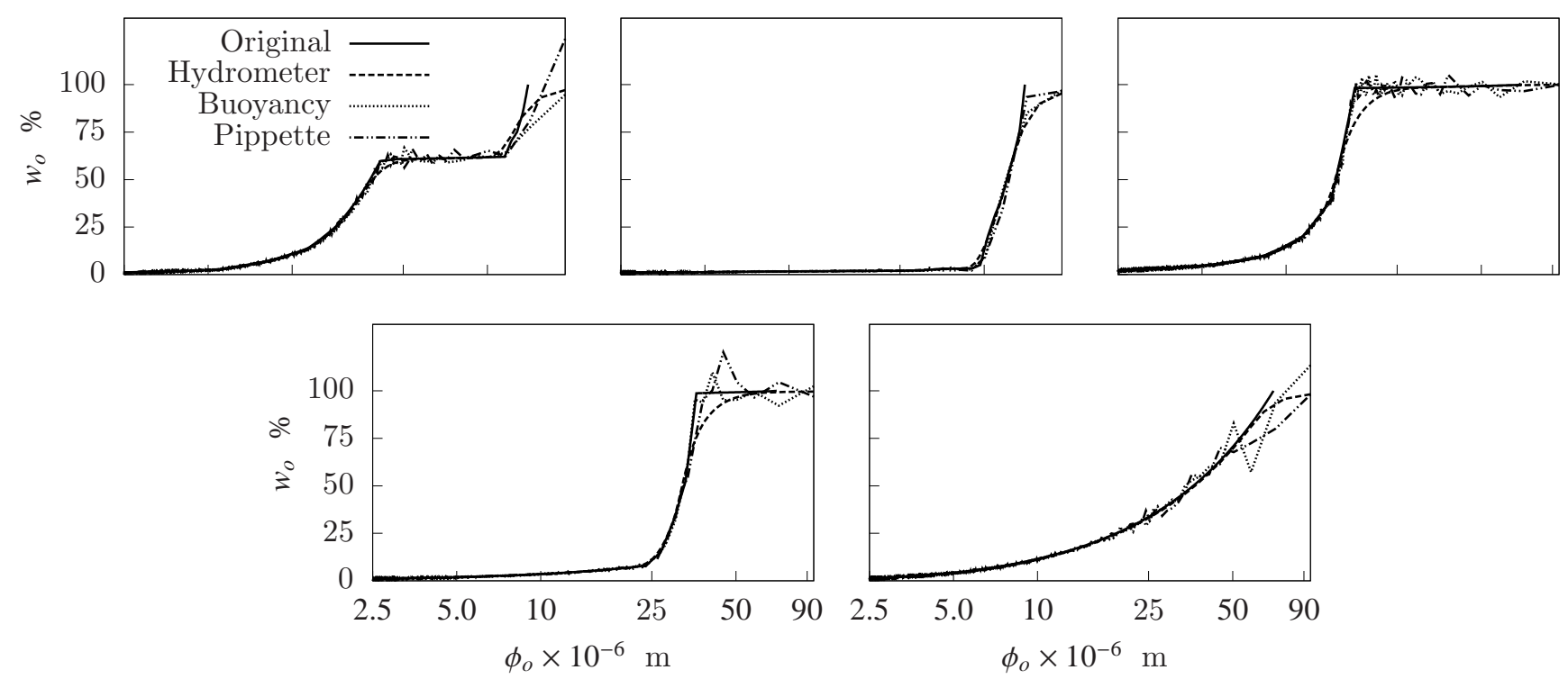

Figure 18: Numerical simulation of granulometry (particle grading size distribution) for samples 1 to 5 (left to right, top to bottom) as would result from the ASTM-D422, buoyancy and pipette tests. Comparison with the original distribution after $40.000 \mathrm{~s}$ of sedimentation.

- Although a large simulation time has been used, a much smaller one can give reasonable results if a small mass and only the initial instants are simulated

Finally, we believe that the use of DEM with sophisticated couplings could be directly applicable to the study of other fluid-particle interaction problems, such as sediment transport, dune evolution or reservoir sedimentation.

\section{Acknowledgements}

R. Bravo and J.L. Pérez-Aparicio were partially supported by the project MICIIN \#BIA-2012-32918. The second researcher used the grant GV BEST/2014/232 for the completion of this work. J. Jaime Gómez-Hernández acknowledges the financial aid from project MINECO CGL2011-23295.

\section{References}

[1] Lowe SA, Sivakumar M. A penalty finite difference model for navier-stokes flow problem in sedimentation basins. Advances in Water Resources 1991;14(6):318-22.

[2] Griffiths C, Dyt C. Accessing sedsim: sedimentary process modelling software. 2004. URL http://www.csiro.au/products/Sedsim.html.

[3] Garzón-Alvarado DA, Galeano C, Mantilla JM. Experimentos numéricos sobre ecuaciones de reacción convección difusión con divergencia nula del campo de velocidad. Revista Internacional de Métodos Numéricos para Cálculo y Diseño en Ingeniería 2010;26(2):69-81.

[4] Jha SK, Bombardelli FA. Theoretical/numerical model for the transport of non-uniform suspended sediment in open channels. Advances in Water Resources 2011;34(5):577-91.

[5] Tetzlaff D, Harbaugh J. Simulating Clastic Sedimentation. Computer Methods in the Geosciences; Springer-Verlag; 1989.

[6] O'Brien JS. Flo-2d: 2-dimensional flood routing model software. 1993. URL http://www.flo-2d.com/.
[7] Krishnaswamy J, Lavine M, Richter DD, Korfmacher K. Dynamic modeling of long-term sedimentation in the yadkin river basin. Advances in Water Resources 2000;23(8):881-92.

[8] Cordero F, Díez P. Xfem+: Una modificación de xfem para mejorar la precisión de los flujos locales en problemas de disfusión con conductividades muy distintas. Revista Internacional de Métodos Numéricos para Cálculo y Diseño en Ingeniería 2010;26(2):121-33.

[9] Cundall P, Strack O. A discrete numerical model for granular assemblies. Geotechnique 1979;29(1):47-65.

[10] Pérez-Aparicio J, Bravo R, Ortiz P. Refined element discontinuous numerical analysis of dry-contact masonry arches. Engineering Structures 2013;48:578-87.

[11] Yeoh G, Tu J. Computational Techniques for Multiphase Flows. Elsevier; 2009.

[12] Bhalla A, Bale R, Griffith B, Patankar N. A unified mathematical framework and an adaptive numerical method for fluidstructure interaction with rigid, deforming, and elastic bodies. Journal of Computational Physics 2013;250:446-76.

[13] Brady J, Bossis G. Stokesian dynamics. Annual review of fluid mechanics 1988;20:111-1157.

[14] Yamanoi M, Pozo O, Maia J. Stokesian dynamics simulation of the role of hydrodynamic interactions on the behavior of a single particle suspending in a newtonian fluid. part 1. 1d flexible and rigid fibers. Journal of Non-Newtonian Fluid Mechanics 2011;166(9):457-68.

[15] Ball RC, Melrose JR. A simulation technique for many spheres in quasi-static motion under frame-invariant pair drag and brownian forces. Physica A 1997;247(1):444-72.

[16] Hager A, Kloss C, Pirker S, Goniva C. Parallel open source cfddem for resolved particle-fluid interaction. Journal of Energy and Power Engineering 2013;9(7):1705-12.

[17] Feng Y, Han K, Owen D. Coupled lattice boltzmann method and discrete element modelling of particle transport in turbulent fluid flows: Computational issues. International Journal for Numerical Methods in Engineering 2007;72(9):1111-34.

[18] Harting J, Chin J, Venturoli M, Coveney P. Large-scale lattice boltzmann simulations of complex fluids: advances through the advent of computational grids. Philosophical transactions of the Royal Society A: Mathematical, Physical and Engineering Sciences 2005;363(1833):1895-915.

[19] Chaumeil F, Crapper M. Using the dem-cfd method to predict brownian particle deposition in a constricted tube. Particuology 
2013;15:94-106

[20] Bravo R, Ortiz P, Pérez-Aparicio J. Incipient sediment transport for non-cohesive landforms by the discrete element method (dem). Applied Mathematical Modelling 2014;38(4):1326-37.

[21] Di-Felice R. The voidage function for fluid-particle interaction systems. International Journal Multiphase Flow 1994;20(1):153-9.

[22] Cunha F. Hydrodynamic dispersion in suspensions. Ph.D. thesis; Department of Applied Mathematics and Theoretical Physics. University of Cambridge; 1995.

[23] Abade G, Cunha F. Computer simulation of particle aggregates during sedimentation. Computer Methods in Applied Mechanics and Engineering 2007;196(45):4597-612.

[24] Bravo R, Pérez-Aparicio J, Laursen T. An energy consistent frictional dissipating algorithm for particle contact problems. International Journal for Numerical Methods in Engineering 2012;92(9):753-81.

[25] Laursen TA. Computational Contact and Impact Mechanics. Springer-Verlag; 2002.

[26] ASTM-D422 . ASTM D422 Standard Test Method for ParticleSize Analysis of Soils. American Society for Testing Materials; 1963.

[27] Bardet J, Young J. Grain-size analysis by buoyancy method. Geotechnical Testing Journal 1997;20(4):481-5.

[28] Tambun R, Motoi T, Shimadzu M, Ohira Y, Obata E. Size distribution measurement of floating particles in the allen region by a buoyancy weighing-bar method. Advanced Powder Technology 2011;22(4):548-52.

[29] Beuselinck L, Govers G, Poesen J, Degraer G, Froyen L. Grainsize analysis by laser diffractometry: comparison with the sievepipette method. Catena 1998;32(3):193-208.

[30] Lambe TW, Whitman RV. Soil Mechanics. John Wiley \& Sons; 1969.

[31] Di-Stefano C, Ferro V, Mirabile S. Comparison between grainsize analyses using laser diffraction and sedimentation methods. Biosystems Engineering 2011;106(2):205-15.

[32] Vdovic N, Obhodas J, Pikelj K. Revisiting the particlesize distribution of soils: Comparison of different methods and sample pre-treatments. European Journal of Soil Science 2010;61(6):854-64.

[33] Nour-Omid B, Wriggers P. A note on the optimum choice for penalty parameters. Communications in Applied Numerical Methods 1987;3(6):581-5.

[34] Bravo R, Pérez-Aparicio J, Laursen T. An enhanced energy conserving time stepping algorithm for frictionless particle contacts. International Journal for Numerical Methods in Engineering 2011;85(11):1415-35.

[35] Heinonen M, Sillanpää S. The effect of density gradients on hydrometers. Measurement Science and Technology $2003 ; 14(5): 625-8$.

[36] Lamb H. Hydrodynamics. Cambridge University Press; 1993.

[37] Lim EW, Wang C. Discrete element simulation for pneumatic conveying of granular material. Particle Technology and Fluidization 2006;52(2):496-509.

[38] Harr M. Mechanics of Particulate Media. A Probabilistic Approach. McGraw-Hill; 1977.

[39] Idelsohn S, nate EO, del Pin F. A lagrangian meshless finite element method applied to fluid-structure interaction problems. Computers and Structures 2003;81(8):655-71.

[40] Wikipedia . Relative density. 2014. http://en.wikipedia.org/wiki/Relative_density.

[41] Munjiza A, Owen DRJ, Bicanic N. Combined finite-discrete element method in transient dynamics of fracturing solids. Engineering computations 1995;12(2):145-74.

[42] Chawla V, Laursen TA. Energy consistent algorithms for frictional contact problems. International Journal for Numerical Methods in Engineering 1998;42(5):799-827.

[43] Di-Felice R. Liquid suspensions of single and binary component solid particles - an overview. China Particuology 2007;5(5):31220.

[44] Reddy KR. Experiment 6: Grain size analysis. 2002. URL 